\title{
A multi-scale map of cell structure fusing protein images and interactions
}

https://doi.org/10.1038/s41586-021-04115-9

Received: 18 June 2020

Accepted: 8 October 2021

Published online: 24 November 2021

Check for updates

\author{
Yue Qin ${ }^{1,2}$, Edward L. Huttlin ${ }^{3}$, Casper F. Winsnes ${ }^{4}$, Maya L. Gosztyla ${ }^{5,6,7}$, Ludivine Wacheul ${ }^{8}$, \\ Marcus R. Kelly', Steven M. Blue ${ }^{5,6,7}$, Fan Zheng', Michael Chen', Leah V. Schaffer', \\ Katherine Licon', Anna Bäckström ${ }^{4}$, Laura Pontano Vaites ${ }^{3}$, John J. Lee', Wei Ouyang ${ }^{4}$, \\ Sophie N. Liu', Tian Zhang ${ }^{3}$, Erica Silva', Jisoo Park', Adriana Pitea', Jason F. Kreisberg', \\ Steven P. Gygi ${ }^{3}$, Jianzhu Ma ${ }^{9}$, J. Wade Harper ${ }^{3}$, Gene W. Yeo ${ }^{2,5,6,7}$, Denis L. J. Lafontaine ${ }^{8}$, \\ Emma Lundberg ${ }^{4,10,11 \bowtie}$ \& Trey Ideker ${ }^{1,2,7,12,13 凶}$
}

\begin{abstract}
The cell is a multi-scale structure with modular organization across at least four orders of magnitude ${ }^{1}$. Two central approaches for mapping this structure-protein fluorescent imaging and protein biophysical association-each generate extensive datasets, but of distinct qualities and resolutions that are typically treated separately ${ }^{2,3}$. Here we integrate immunofluorescence images in the Human Protein Atlas $^{4}$ with affinity purifications in BioPlex ${ }^{5}$ to create a unified hierarchical map of human cell architecture. Integration is achieved by configuring each approach as a general measure of protein distance, then calibrating the two measures using machine learning. The map, known as the multi-scale integrated cell (MuSIC 1.0), resolves 69 subcellular systems, of which approximately half are to our knowledge undocumented. Accordingly, we perform 134 additional affinity purifications and validate subunit associations for the majority of systems. The map reveals a pre-ribosomal RNA processing assembly and accessory factors, which we show govern rRNA maturation, and functional roles for SRRM1 and FAM120C in chromatin and RPS3A in splicing. By integration across scales, MuSIC increases the resolution of imaging while giving protein interactions a spatial dimension, paving the way to incorporate diverse types of data in proteome-wide cell maps.
\end{abstract}

Eukaryotic cells consist of large components, such as organelles, which recursively factor into smaller components, such as condensates and protein complexes, forming an intricate multi-scale structure ${ }^{6}$. Fundamental techniques for mapping subcellular structure are protein imaging and biophysical association, each of which has been extensively automated. In particular, advances in confocal microscopy and immunofluorescence have made it possible to scan the distribution of proteins in situ within single cells ${ }^{2}$. By combining these techniques with a library of antibodies, the Human Protein Atlas (HPA) has embarked on systematic studies to position human proteins into subcellular compartments ${ }^{4}$. As a parallel approach to cell mapping, mass spectrometry (MS) has been powerfully combined with affinity purification (AP-MS) and proximity-dependent labelling to enable rapid measurement of protein-protein associations ${ }^{3}$. Using AP-MS, the BioPlex project is generating comprehensive interaction maps for most human proteins ${ }^{5}$.

Given these efforts, a key question is how imaging and biophysical association should be combined to inform cell structure. We reasoned that the two platforms provide complementary measures of protein location, albeit of vastly different characters. Images position proteins relative to cellular landmarks such as the nucleus, whereas biophysical associations position proteins relative to nearby proteins. In both cases, such positioning has become increasingly quantitative due, in part, to the ability of machine learning systems to recognize complex patterns in data ${ }^{7,8}$.

Here we demonstrate a machine learning approach in which protein imaging and biophysical association are integrated to create a unified map of subcellular components (Fig. 1). First, we use neural networks to project proteins into a small number of dimensions on the basis of imaging or biophysical association. Once protein coordinates have been determined for each platform, pairwise distances among proteins are calibrated and combined to reveal assemblies at different scales, from the very small (less than $50 \mathrm{~nm}$ ) to the very large (more than $1 \mu \mathrm{m}$ ).

\section{Protein position and distance in two ways}

We assembled a matched dataset of immunofluorescence images from $\mathrm{HPA}^{4}$ and AP-MS data from BioPlex ${ }^{5}$. Both resources are partially based

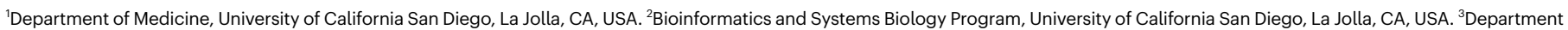
of Cell Biology, Harvard Medical School, Boston, MA, USA. ${ }^{4}$ Science for Life Laboratory, School of Engineering Sciences in Chemistry, Biotechnology and Health, KTH Royal Institute of

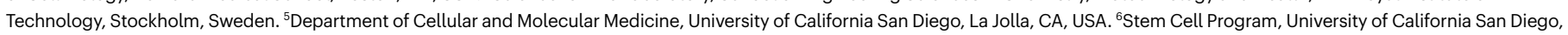
La Jolla, CA, USA. Institute for Genomic Medicine, University of California San Diego, La Jolla, CA, USA. ${ }^{8}$ RNA Molecular Biology, Fonds de la Recherche Scientifique (F.R.S./FNRS), Université Libre de Bruxelles (ULB), Charleroi-Gosselies, Belgium. ${ }^{9}$ Institute for Artificial Intelligence, Peking University, Beijing, China. ${ }^{10}$ Department of Genetics, Stanford University, Stanford, CA, USA.

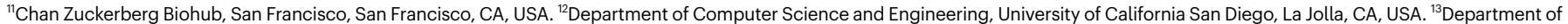
Bioengineering, University of California San Diego, La Jolla, CA, USA. ${ }^{凶}$-mail: emma.lundberg@scilifelab.se; tideker@ucsd.edu 


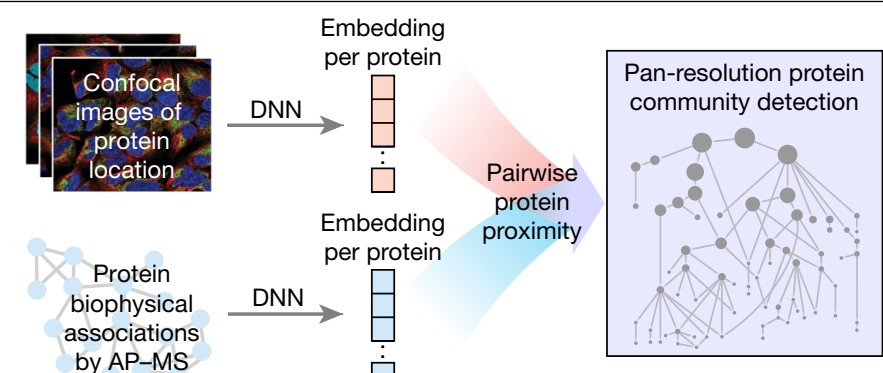

Fig. 1 Overview of data fusion strategy. Protein images and interaction data are analysed to generate neural network embeddings for each protein. These embeddings reveal communities of proximal proteins at multiple resolutions to create a multi-scale integrated map of the cell. DNN, deep neural network.

on human embryonic kidney (HEK293-derived) cells, yielding 661 proteins with compatible imaging (1,451 images including replicates) (Extended Data Fig. 1a-c) and biophysical association data (291 proteins affinity-tagged as 'baits', 370 as interacting 'preys') (Supplementary Table1). These proteins covered a wide distribution of subcellular locations similar to that seen for all human proteins (Extended Data Fig. 1d). Other proteins in HPA and BioPlex were measured in differing cell types that did not align; thus, we focused on the common HEK293-derived context for prototyping our approach.
We next used deep neural networks to embed each protein on the basis of its immunofluorescence and AP-MS data. An embedding is a low-dimensional representation of a complex input, in which each data point (here a protein) is assigned coordinates in the reduced dimensions. Much machine learning research has focused on creating a good embedding, in which similar inputs (here proteins with similar subcellular distributions or interactions) are close in the embedded space ${ }^{9}$. For image embedding we used DenseNet ${ }^{7}$, a convolutional neural network with superior performance in capturing protein locations relative to counter-stained cellular landmarks (Extended Data Fig. 2a-c). Similarly, the node2vec neural network ${ }^{8}$ was used to embed each protein using its extended AP-MS interaction neighbourhood (Extended Data Fig. $2 \mathrm{~d}-\mathrm{g})$.

We then computed protein-protein distances for all protein pairs, separately in immunofluorescence and AP-MS embeddings. The closest pairs measured by one technique were enriched for pairs close in the other, showing that imaging and AP-MS share substantial information (Extended Data Fig. 3a, b). To calibrate distances in the embeddings to physical distances in cells, we assembled a reference set of subcellular components with known or estimated diameters, from protein complexes of less than $20 \mathrm{~nm}$ to organelles of more than $1 \mu \mathrm{m}$ (Extended Data Fig. 3c, Supplementary Table 2, Supplementary Methods). With these curated diameters as training labels, we taught a supervised machine learning model (random forest regression) to estimate the distance of any protein pair directly from its coordinates in the immunofluorescence and AP-MS embeddings (Extended Data Fig. 3d, e).

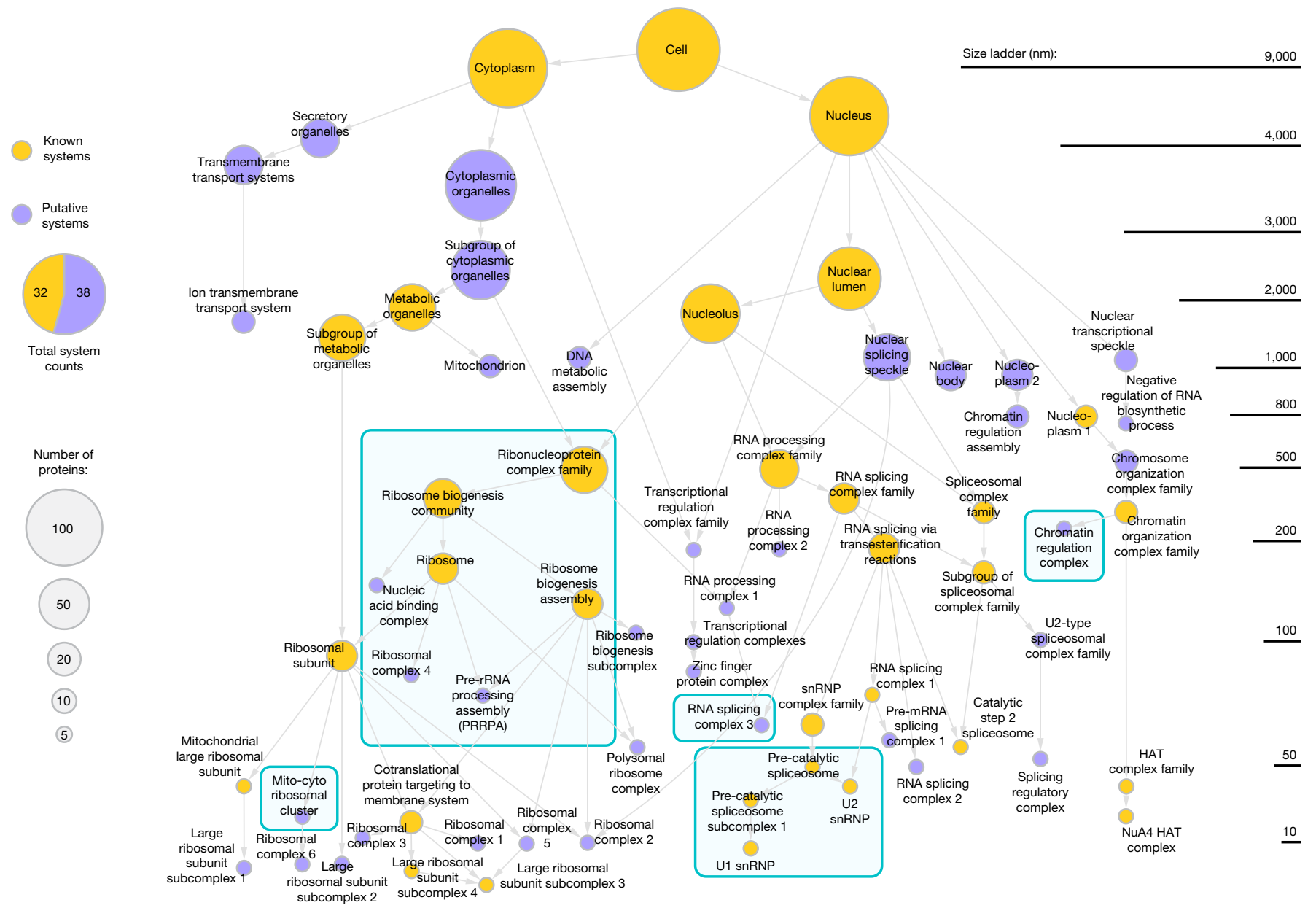

Fig. 2 The multi-scale integrated cell. Nodes indicate systems; arrows indicate containment of lower system by upper. Node size, number of system proteins. Node colour, known (gold) versus novel (purple). Teal boxes denote systems detailed in the text and figures. Elevation of system (size ladder) determined by predicted diameter. 


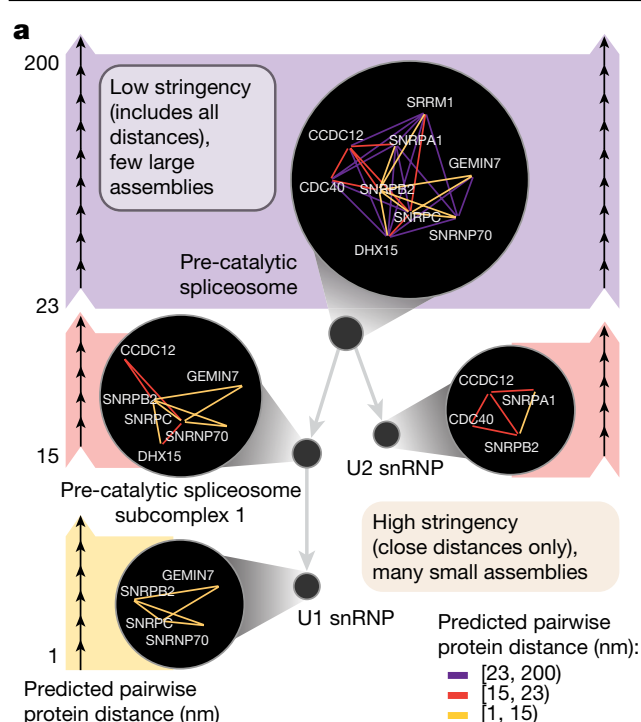

d

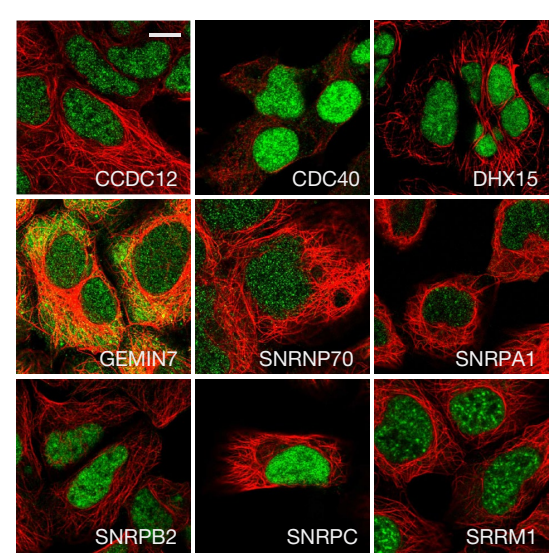

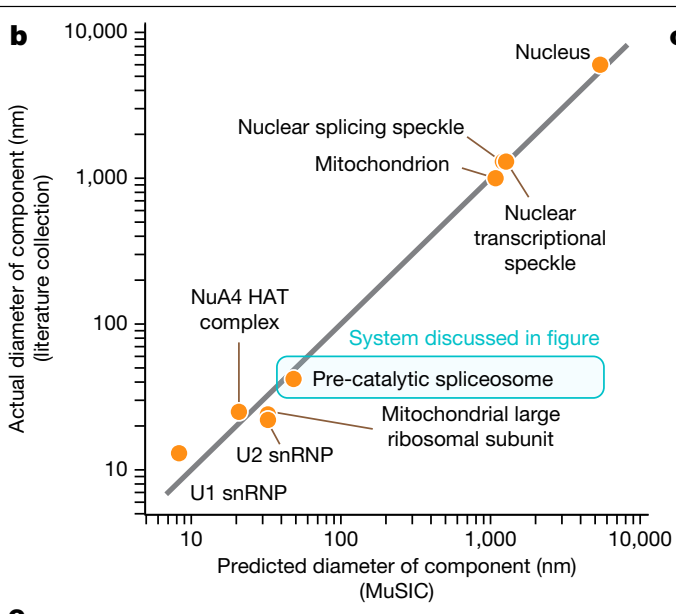
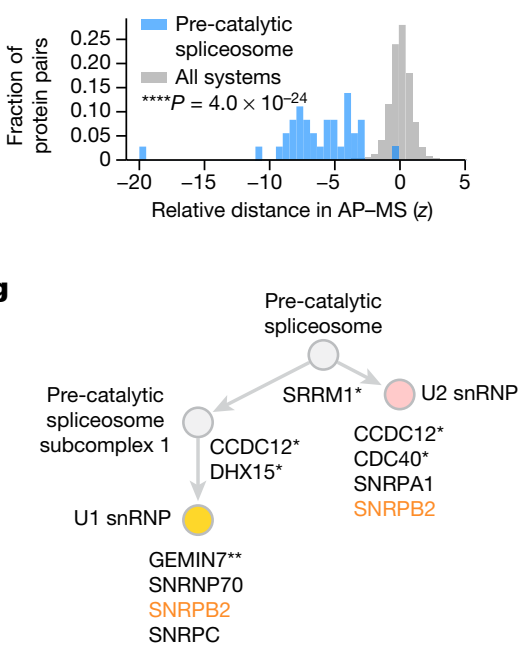

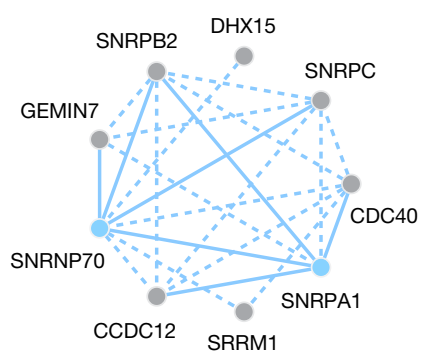

BioPlex bait

- AP-MS interaction AP-MS interaction (path-length $=2$ )

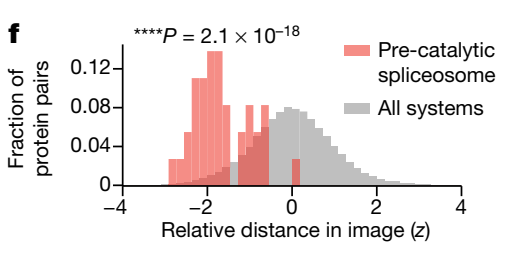

Fig. 3 | MuSIC captures subcellular components and diameters.

a, Hierarchical community detection. As distance threshold increases (bottom to top), strong communities (systems) are detected first, then expanded to include moderate-to-weak associations. Dark circles, systems; edge colours, association stringency.b, Predicted versus actual diameter of components detailed in literature and not used for calibration. c, Biophysical interaction data for pre-catalytic spliceosome. AP-MS interaction (path-length =2) indicates protein pairs that interact with common affinity-tagged bait(s) outside the complex.d, Same proteins immunostained (green) with cytoskeleton counterstain (red). Scale bar, $10 \mu \mathrm{m}$.e, Histogram of protein-

\section{A multi-scale map of subcellular systems}

We analysed all distances among the 661 proteins to identify communities of proteins in close mutual proximity, suggesting distinct components (Fig. 2). Communities were identified at multiple resolutions, starting with those that form at the smallest protein-protein distances, then progressively relaxing the distance threshold (multi-scale community detection ${ }^{10}$ Extended Data Fig. 4a, Supplementary Methods). Communities at smaller distances were contained, in full or part, inside larger communities as the threshold was relaxed, yielding a structural hierarchy (Fig. 3a). The sensitivity of community detection was tuned for best concordance with two independent datasets: protein interactions reported in the Human Cell Map ${ }^{11}$ using proximity biotinylation, also in HEK293 cells; and patterns of gene co-essentiality in the Cancer Cell Dependency Map ${ }^{12}$. Significant agreement with independent datasets was observed for a wide range of community detection parameters and for both small and large communities (Extended Data Fig. 4b, e). The final hierarchy, MuSIC 1.0, contained 69 protein communities

protein distances in AP-MS embedding ( $z$-scores). Blue, pre-catalytic spliceosome; grey, all protein pairs. One-sided Mann-Whitney $U$ test.f, As in e for image rather than AP-MS data. Red, pre-catalytic spliceosome; grey, all protein pairs. g, Hierarchy of spliceosome systems in MuSIC (left) versus 3D structural model (right; Protein Data Bank 6QX $9^{14}$ ). ${ }^{*}$ indicates a pre-catalytic spliceosome protein ${ }^{13}$ captured by MuSIC but not included in structural model; ** indicates a protein important for small nuclear ribonucleoprotein (snRNP) assembly. Proteins are assigned the same colours in both maps. SNRPB2 (orange) is in both U1 and U2 subunits in MuSIC, as suggested previously ${ }^{30}$; the structural model places it in U2 only.

representing putative subcellular systems organized by 87 hierarchical containment relationships (Fig. 2, Supplementary Table 3). Sixteen systems were contained within multiple larger ones, suggesting multiple subcellular locations or pleiotropy. Approximately $46 \%$ had a substantial overlap with cellular components documented in Gene Ontology; we annotated the remaining 54\% as putatively novel (Fig. 2).

Physical sizes of MuSIC systems were estimated from their pairwise protein distances (Fig. 2) and compared to known diameters of nine well-characterized cellular components not used earlier in calibration (Fig. 3b, Supplementary Table 4). One of these was the pre-catalytic spliceosome, for which support from both immunofluorescence and AP-MS data (Fig. 3c-f) had induced a protein community of $48 \mathrm{~nm}$ ( $95 \%$ prediction interval $[26,90])$, in agreement with its published diameter of $42 \mathrm{~nm}^{13,14}$ (Fig. 3a, g). Within this community, the analysis resolved smaller $U 1$ and $U 2$ subunits (U1: $8 \mathrm{~nm}, 95 \%$ prediction interval $[4,15]$; $\mathrm{U} 2: 33 \mathrm{~nm}, 95 \%$ prediction interval $[17,61])$, again in agreement with the arrangement and distances measured by cryo-electron microscopy (Fig. 3g). For all nine components, estimated diameters were very 
a

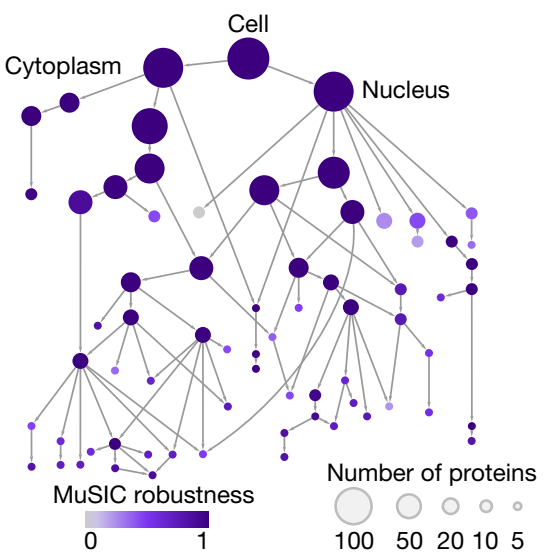

d

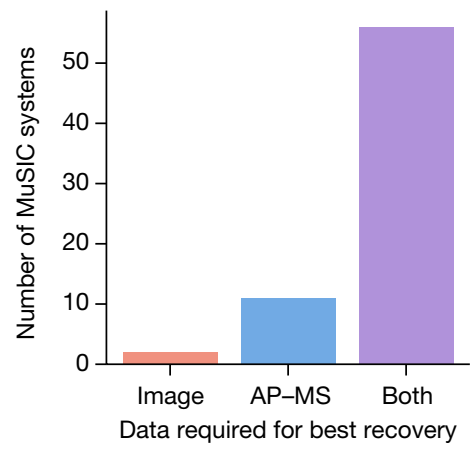

b

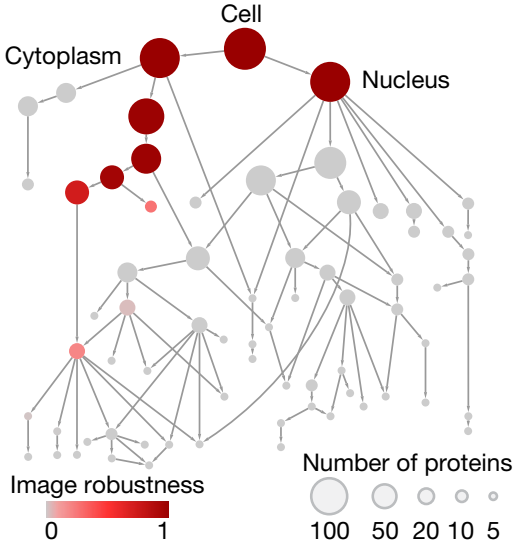

e

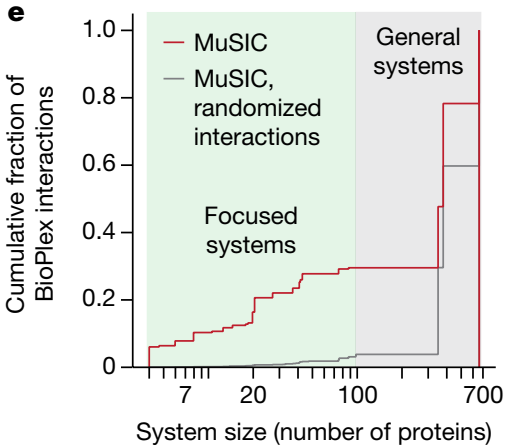

c
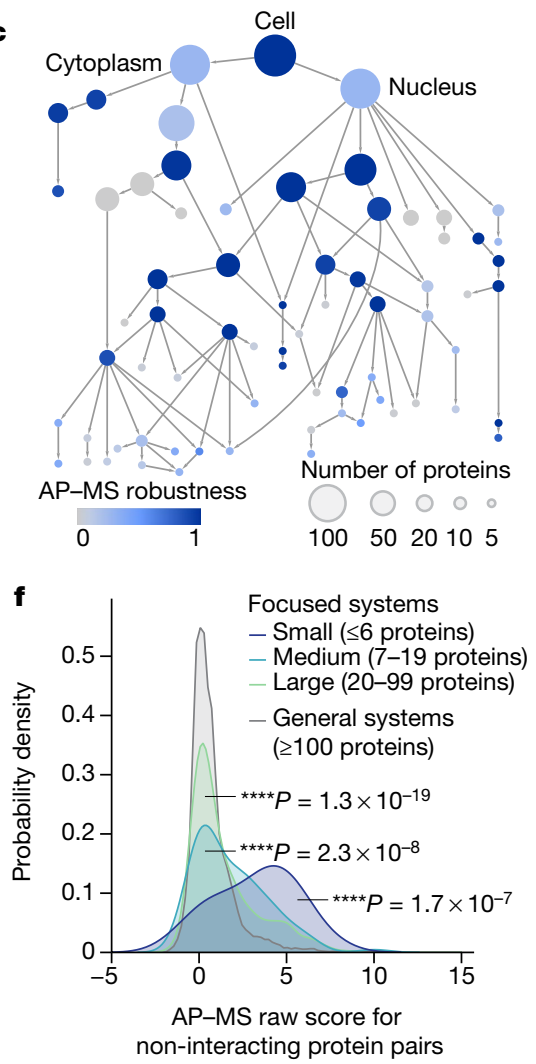

Fig. 4 | Different data informs different scales of information. a-c, MuSIC map from Fig. 2, coloured with system robustness when built using imaging and AP-MS data (full MuSIC (a)), imaging only (b) or AP-MS only (c). d, Number of systems for which highest robustness comes with imaging, AP-MS or both types. e, Cumulative fraction of AP-MS interactions within MuSIC systems (red) versus random protein pairs (grey; 1,000 randomizations).f, Distribution of AP-MS scores for protein pairs not labelled as interacting by BioPlex. $P$ values calculated against general systems (at least 100 proteins); one-sided Mann-Whitney $U$ test. close to actual measurements from the literature (Fig. 3b), validating that MuSIC captures and sizes biological systems across a wide range of scales.

\section{MuSIC needs and informs both data types}

We found that the majority of systems were robust to minor disruptions in data (Fig. 4a, jackknife resampling, Supplementary Methods). By contrast, alternative MuSIC maps constructed with only one data type dropped numerous systems. Immunofluorescence-only maps tended to identify large systems such as organelles but falter for small subcomponents such as protein complexes, whereas AP-MS maps had the opposite behaviour (Fig. 4b-d). Notably, 30\% of AP-MS interactions fell within focused systems of fewer than 100 proteins (Fig. 4e), validating and providing location context for the interaction. Such context also increases the sensitivity of interaction detection: focusing on protein pairs not reported to interact in the previous BioPlex study ${ }^{5}$, pairs in smaller systems nonetheless had stronger AP-MS scores than pairs in larger systems $(P<0.0001$; Fig. $4 \mathrm{f})$, suggesting new bona fide physical interactions.

\section{Global validation of MuSIC by new AP-MS}

Of the $661 \mathrm{MuSIC}$ proteins, 370 had not yet been affinity-tagged as baits in $\mathrm{AP}-\mathrm{MS}$ experiments. Rather, they had appeared in the list of prey proteins isolated by another affinity-tagged protein. As an immediate means of validating candidate systems, we affinity-tagged 134 former prey proteins and performed $\mathrm{AP}-\mathrm{MS}$, resulting in the identification of
339 physical interactions (Supplementary Table 1). Forty-four MuSIC systems were specifically enriched for new interactions (64\%; false discovery rate $($ FDR $)<0.1$ ( Fig. 5a), including 23 putative candidates.

\section{Ribosomal systems at multiple scales}

Among candidates validated by the additional AP-MS data was a seven-protein assembly with an estimated diameter of $81 \mathrm{~nm}$ (95\% prediction interval $[43,151])$. We tentatively named this system 'pre-ribosomal RNA processing assembly' (PRRPA) on the basis of established pre-rRNA roles for two of its proteins ${ }^{15,16}$ (NVL, RPL13A), support from genetic screens ${ }^{17}$ (KRI1, NOC2L) and orthology to a pre-rRNA factor in yeast ${ }^{18}$ (REXO4). These proteins formed a system due to image similarity, with nucleolar localizations, and similarity of AP-MS network neighbourhoods (Fig. 5b, c, Extended Data Fig. 5a). Our new affinity purifications targeted five PRRPA proteins, and recovered interacting partners highly specific to this system (Fig. 5c, Extended Data Fig. 5b). To explore the function of PRRPA in pre-rRNA processing, we used small interfering RNAs (siRNAs) to knock down each protein; all knockdowns perturbed ribosomal RNA maturation to some extent (Extended Data Fig. 5c-i). We then used RNA immunoprecipitation and quantitative PCR (RIP- $q$ PCR) to find that these proteins bind 45 S pre-rRNA, again supporting a pre-rRNA processing role (Fig. $5 \mathrm{~d}$ ).

We also examined the larger-scale system containing PRRPA, 'ribosome biogenesis community' ( $347 \mathrm{~nm}, 95 \%$ prediction interval [186, 646]). This system contained additional proteins not associated with ribosome biogenesis (Extended Data Fig. 6a), seven of which we knocked down with targeted Dicer-substrate siRNAs (DsiRNAs). All 


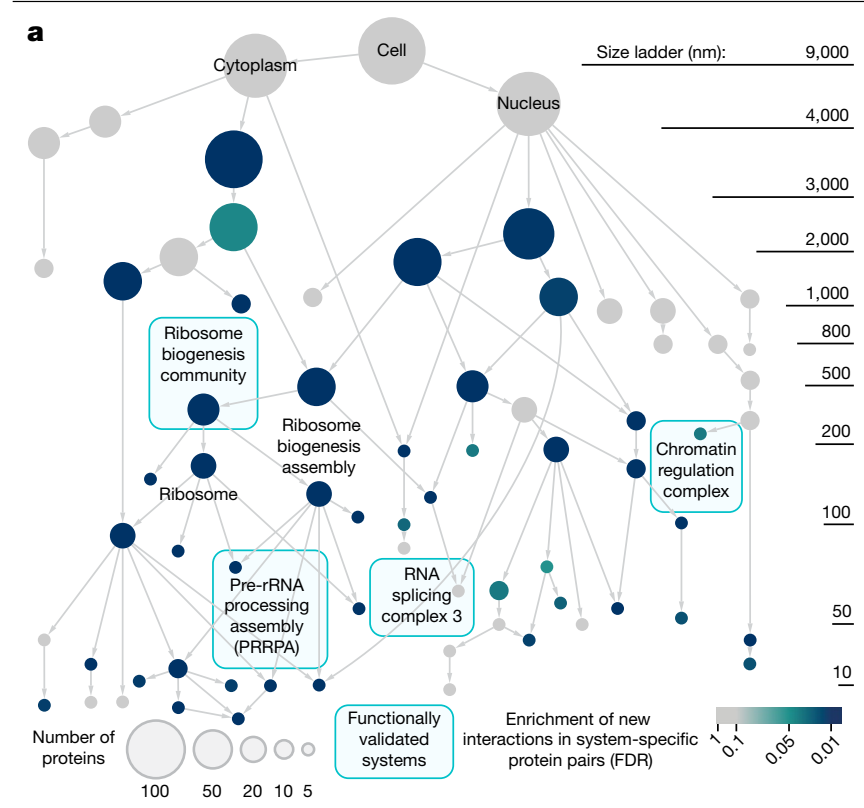

e

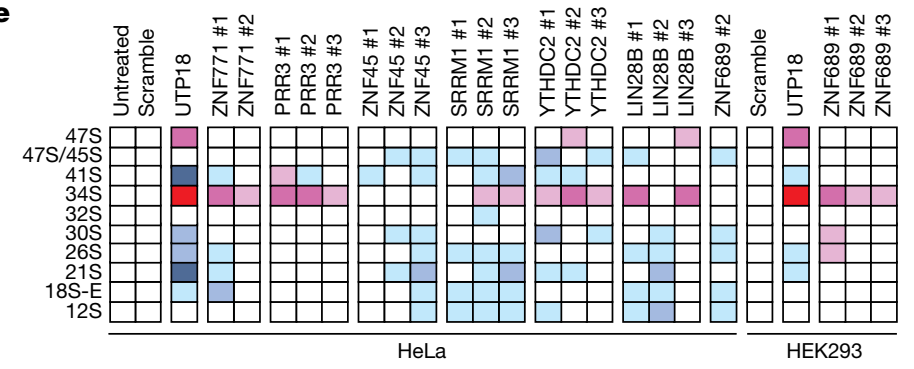

$<25 \% \square 25-50 \% \square 50-67 \% \square 67-125 \% \square 125-175 \% \square 175-400 \% \square>400 \%$

$\mathbf{f}$

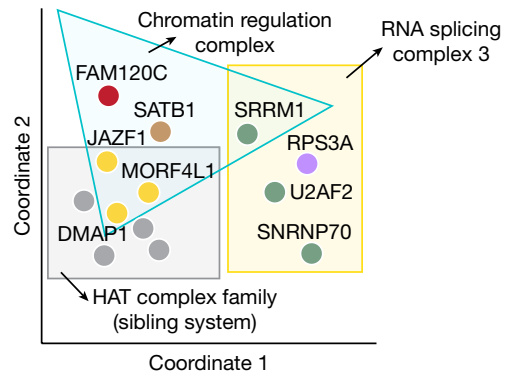

Previous function:

H4 HAT

Chromatin organizer

Splicing factor

Ribosomal protein

Unknown function b
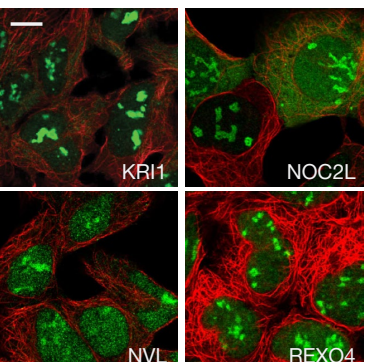

NOC2L
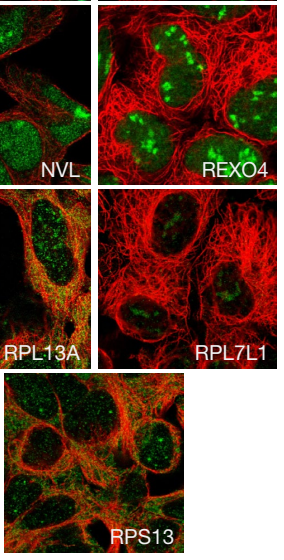

g

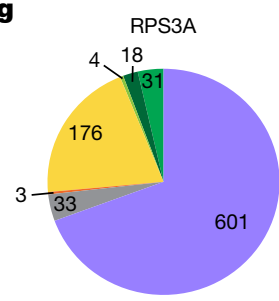

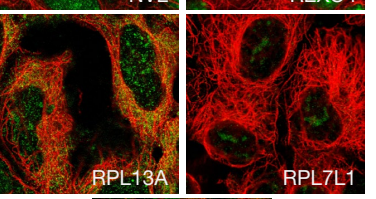

c

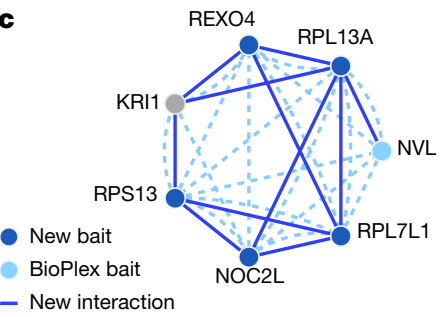

- New interaction

BioPlex interaction (path-length $=2$ )

d

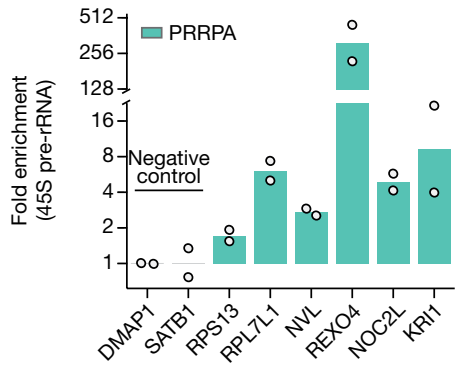

Canonical Canonical splicing ribosomal regulators proteins

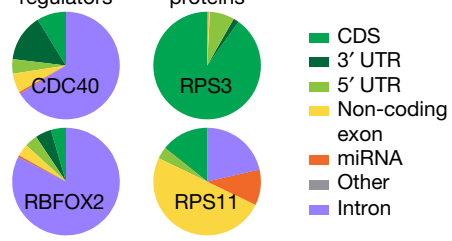

$\mathbf{h}$

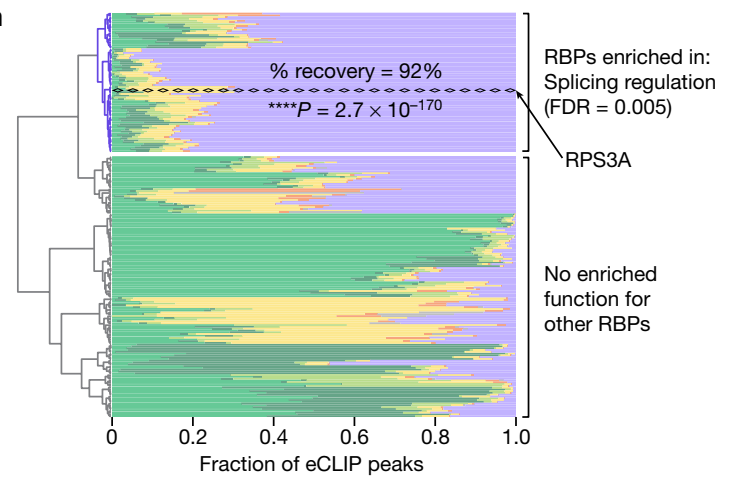

Independent silencers (\#1-3) were highly consistent.f, Two-dimensional projection (spring embedding) of distances among proteins in chromatin regulation and splicing complexes. g, Pie charts categorize significant eCLIP peaks by genomic region (coloured slices). CDS, coding sequence; miRNA, microRNA; UTR, untranslated region. h, Clustering of RPS3A eCLIP profile (dashed line) with 223 eCLIP profiles ${ }^{25}$. Proteins robustly clustering with RPS3A (1,000 jackknife resamplings) enrich for splicing regulators (hypergeometric test, Benjamini-Hochberg correction). Colour consistent with g. RBP, RNA-binding protein. seven had effects on pre-rRNA processing, stratified by the specific pre-rRNA affected (Fig. 5e, Extended Data Fig. 6b, c). Three of these proteins were targeted in our new AP-MS experiments (LIN28B, PRR3, ZNF689); each was shown to bind a substantial number of proteins within this same community (Extended Data Fig. 6d).

Another notable finding within ribosomal systems was abundant cross-talk between cytoplasmic and mitochondrial ribosomes ('mito-cyto ribosomal cluster'; $20 \mathrm{~nm}, 95 \%$ prediction interval $[11,38]$ ) (Extended Data Fig. 6e-h). Several of these proteins were tagged in the new AP-MS experiments (two cytoribosomal, two mitoribosomal), recovering four new physical interactions between cytoplasmic and mitochondrial factors (Extended Data Fig. 6i). Such cross-talk may have a role in mitoribosome biogenesis, a poorly understood process ${ }^{19}$.

\section{Chromatin and splicing}

SRRM1 is an established splicing factor ${ }^{20}$ that, in addition to its canonical placement in 'RNA splicing complex 3' (71 nm, 95\% prediction interval 
$[38,133])$, participated in additional systems that were unexpected. 'Chromatin regulation complex' (211 nm, 95\% prediction interval [113, 393]) included three histone acetyltransferases (HATs) (DMAP1,JAZF1 and MORF4L1 ${ }^{21}$ ) and SATB1, which remodels chromatin through HAT recruitment ${ }^{22}$ (Fig. 5f, Extended Data Fig. 7a, b). These functions suggested that SRRM1 and FAM120C, the remaining proteins in this system, also regulate chromatin. In support of this, we found that SRRM1 and FAM120C strongly associate with chromatin by in situ fractionation (Extended Data Fig. 7c).

Returning to RNA splicing complex 3, this system brought SRRM1 and other splicing factors $\left(\mathrm{SNRNP70}{ }^{23}, \mathrm{U} 2 \mathrm{AF}^{24}\right.$ ) together with a ribosomal protein that was not previously associated with major RNA splicing $\left(\right.$ RPS3A $\left.^{21}\right)$ (Extended Data Fig. 7d, e). However, analysis of published transcriptomic profiles ${ }^{25}$ indicated that knockdown of RPS3A had very similar transcriptional effects to knockdown of these splicing factors (Extended Data Fig. 7f, g). To test for a role in splicing, we subjected RPS3A to an enhanced ultraviolet cross-linking and immunoprecipitation assay ${ }^{26}$ (eCLIP, Extended Data Fig. 7h), which identifies and characterizes RNA transcripts bound by a protein. Indeed, RPS3A bound to many intronic RNA sequences (601 eCLIP peaks) (Supplementary Table 5) with a pattern very similar to that of canonical splicing regulators (Fig. 5g). Moreover, when clustering the RPS3A profile with 223 eCLIP profiles from the public domain ${ }^{25}$, RPS3A robustly clustered with canonical splicing regulators ( $92 \%$ recovery in jackknife resampling) (Fig.5h), providing further support for an alternative role of this protein in splicing regulation.

\section{Discussion}

In classical image analysis, protein proximity is measured by fluorescently labelling multiple proteins in the same image ${ }^{27}$, a combinatorial process that is difficult to scale. Here we have developed a systematic means of measuring proximity through neural network embeddings of each protein. In turn, systematic accumulation of protein proximities moves us from a fixed list of predefined subcellular components to an open approach in which components are defined by inherent structure in the imaging data. Such analysis also integrates with other types of information, demonstrated here with $\mathrm{AP}-\mathrm{MS}$, and recovers components at multiple scales (Fig. 3b), including novel systems that can be physically and functionally validated (Fig. 5). Although imaging research is accustomed to thinking about physical sizes and intracellular distances, the notion that protein interactions provide a complementary measure of intracellular distance is, to our knowledge, new to this study.

Although nearly a third of AP-MS interactions link proteins within a focused system of fewer than 100 proteins, more than two thirds do not (Fig. 4e, Extended Data Fig. 8a). Such discrepancies may indicate transient protein interactions. Alternatively, discrepancies might derive from errors or biases, such as the fact that immunofluorescence detects endogenous proteins whereas AP-MS detects overexpressed tagged proteins. Some disagreement between data types can be tolerated, such as the correct assignment of GEMIN7 and SNRNP70 to the U1 snRNP (Fig. 3g), despite only a partial overlap in their images (Extended Data Fig. 8b). Here, correct assignment was facilitated by physical interaction from AP-MS.

Systems in MuSIC reside at multiple scales, bridging and exceeding the ranges of immunofluorescence and AP-MS (Fig. 4a-d). Here the scale of a component is determined by the estimated nanometre proximities among its members; this measurement of scale only partially correlates with the component's number of proteins. Analysis of protein proximities at broad scale identified the pre-catalytic spliceosome, whereas decreasing the distance threshold recovered smaller subcomponents, the U1 and U2 snRNPs (Fig. 3a, g). As physical proximity increases, one would expect the same for functional association. To this point, gene co-essentiality-a measure of joint function ${ }^{28}$-was strongest among genes in the same small systems, weaker within larger systems that contain them and near zero for unrelated genes (Extended Data Fig. 8c, d). Components at different scales map naturally to different types of assays for functional exploration. For example, we used 28S/18S rRNA ratio as a general readout affected by proteins in the ribosome biogenesis community. More specific probes implicated specific subfunctions, such as the binding of a protein to 45 S pre-rRNA (suggesting early-stage ribosome biogenesis) (Fig. 5d) or changes in 34S pre-rRNA that result from protein knockdown (suggesting maturation defect associated with small-subunit processome ${ }^{17}$ ) (Fig. 5e). We expect future validation of MuSIC systems to draw from a range of functional assays at the molecular, pathway and cellular level.

As the map is developed to cover all human proteins, key questions relate to cellular heterogeneity and dynamics; for example, whether it is preferable to work towards a unified map of subcellular components or to create separate maps cataloguing different cell types and states. An attractive middle road may be to create a small library of reference maps for major cell types, with context-specific differences indicated as annotations. Here, we focused on HEK293-derived cells, a widely used model for gaining general biological insights ${ }^{4,5,11}$. Previous studies have shown that approximately $70 \%$ of proteins have consistent localization across cell lines ${ }^{4}$ and about $50 \%$ maintain their physical interactions ${ }^{29}$; thus, we expect that the current map will partially generalize to other contexts, with attention paid to communities prone to dynamics. Notably, the proteins of many MuSIC systems are co-regulated in expression across diverse cell types (Extended Data Fig. 8e), suggesting that these systems are indeed relevant to other contexts.

Finally, we note the synergy achieved in integrating HPA and BioPlex, two large-scale mapping efforts that might have progressed independently. Such coordination should continue and encompass collaborative dataset design; for instance, by adopting common cell lines and proteins targeted across projects. Furthermore, new protein systems might arise with the inclusion of additional data modalities, such as proximity-dependent labelling, cross-linking mass spectrometry or cryo-electron microscopy. It will be interesting to explore synergies among these platforms, all of which might be calibrated to measure molecular distances and, in turn, contribute to maps of the multi-scale cell.

\section{Online content}

Any methods, additional references, Nature Research reporting summaries, source data, extended data, supplementary information, acknowledgements, peer review information; details of author contributions and competing interests; and statements of data and code availability are available at https://doi.org/10.1038/s41586-021-04115-9.

1. Harold, F. M. Molecules into cells: specifying spatial architecture. Microbiol. Mol. Biol. Rev. 69, 544-564 (2005).

2. Mori, H. \& Cardiff, R. D. Methods of immunohistochemistry and immunofluorescence: converting invisible to visible. In The Tumor Microenvironment, Methods in Molecular Biology Vol. 1458 (eds Ursini-Siegel, J. \& Beauchemin, N.) 1-12 (Humana Press, 2016).

3. Aebersold, R. \& Mann, M. Mass-spectrometric exploration of proteome structure and function. Nature 537, 347-355 (2016).

4. Thul, P. J. et al. A subcellular map of the human proteome. Science 356, eaal3321 (2017).

5. Huttlin, E. L. et al. Architecture of the human interactome defines protein communities and disease networks. Nature 545, 505-509 (2017).

6. Schaffer, L. V. \& Ideker, T. Mapping the multiscale structure of biological systems. Cell Syst. 12, 622-635 (2021).

7. Ouyang, W. et al. Analysis of the Human Protein Atlas Image Classification competition. Nat. Methods 16, 1254-1261 (2019).

8. Grover, A. \& Leskovec, J. node2vec: scalable feature learning for networks. In KDD '16: Proc. 22nd ACM SIGKDD International Conference on Knowledge Discovery and Data Mining 855-864 (2016).

9. Goodfellow, I., Bengio, Y., Courville, A. \& Bengio, Y. Deep Learning Vol. 1 (MIT Press, 2016).

10. Fortunato, S. \& Hric, D. Community detection in networks: a user guide. Phys. Rep. 659, 1-44 (2016).

11. Go, C. D. et al. A proximity-dependent biotinylation map of a human cell. Nature $\mathbf{5 9 5}$, 120-124 (2021)

12. Meyers, R. M. et al. Computational correction of copy number effect improves specificity of CRISPR-Cas9 essentiality screens in cancer cells. Nat. Genet. 49, 1779-1784 (2017). 


\section{Article}

13. Deckert, J. et al. Protein composition and electron microscopy structure of affinity-purified human spliceosomal B complexes isolated under physiological conditions. Mol. Cell. Biol. 26, 5528-5543 (2006).

14. Charenton, C., Wilkinson, M. E. \& Nagai, K. Mechanism of 5 ' splice site transfer for human spliceosome activation. Science 364, 362-367 (2019).

15. Yoshikatsu, Y. et al. NVL2, a nucleolar AAA-ATPase, is associated with the nuclear exosome and is involved in pre-rRNA processing. Biochem. Biophys. Res. Commun. 464 780-786 (2015).

16. Chaudhuri, S. et al. Human ribosomal protein L13a is dispensable for canonical ribosome function but indispensable for efficient rRNA methylation. RNA 13, 2224-2237 (2007).

17. Tafforeau, L. et al. The complexity of human ribosome biogenesis revealed by systematic nucleolar screening of pre-rRNA processing factors. Mol. Cell 51, 539-551 (2013).

18. Eppens, N. A. et al. Deletions in the S1 domain of Rrp5p cause processing at a novel site in ITS1 of yeast pre-rRNA that depends on Rex4p. Nucleic Acids Res. 30, 4222-4231 (2002).

19. De Silva, D., Tu, Y.-T., Amunts, A., Fontanesi, F. \& Barrientos, A. Mitochondrial ribosome assembly in health and disease. Cell Cycle 14, 2226-2250 (2015).

20. Blencowe, B. J. et al. The SRm160/300 splicing coactivator subunits. RNA 6, 111-120 (2000).

21. The UniProt Consortium. UniProt: a worldwide hub of protein knowledge. Nucleic Acids Res. 47, D506-D515 (2019).

22. Pavan Kumar, P. et al. Phosphorylation of SATB1, a global gene regulator, acts as a molecular switch regulating its transcriptional activity in vivo. Mol. Cell 22, 231-243 (2006).
23. Pomeranz Krummel, D. A., Oubridge, C., Leung, A. K. W., Li, J. \& Nagai, K. Crystal structure of human spliceosomal U1 snRNP at 5.5 A resolution. Nature $\mathbf{4 5 8}, 475-480$ (2009).

24. Fleckner, J., Zhang, M., Valcárcel, J. \& Green, M. R. U2AF65 recruits a novel human DEAD box protein required for the U2 snRNP-branchpoint interaction. Genes Dev. 11, 1864-1872 (1997).

25. Van Nostrand, E. L. et al. A large-scale binding and functional map of human RNA-binding proteins. Nature 583, 711-719 (2020).

26. Van Nostrand, E. L. et al. Robust, cost-effective profiling of RNA binding protein targets with single-end enhanced crosslinking and immunoprecipitation (seCLIP). In mRNA Processing, Methods in Molecular Biology Vol. 1648 (ed. Shi, Y.) 177-200 (Humana Press, 2017).

27. Stryer, L. Fluorescence energy transfer as a spectroscopic ruler. Annu. Rev. Biochem. 47 819-846 (1978).

28. Wang, T. et al. Gene essentiality profiling reveals gene networks and synthetic lethal interactions with oncogenic Ras. Cell 168, 890-903 (2017).

29. Huttlin, E. L. et al. Dual proteome-scale networks reveal cell-specific remodeling of the human interactome. Cell 184, 3022-3040 (2021).

30. Williams, S. G. \& Hall, K. B. Human U2B" protein binding to snRNA stemloops. Biophys. Chem. 159, 82-89 (2011).

Publisher's note Springer Nature remains neutral with regard to jurisdictional claims in published maps and institutional affiliations.

(c) The Author(s), under exclusive licence to Springer Nature Limited 2021 


\section{Reporting summary}

Further information on research design is available in the Nature Research Reporting Summary linked to this paper.

\section{Data availability}

A web portal is available at http://nrnb.org/music with links to all major resources used for this study. These include the MuSIC map (https:// doi.org/10.18119/N9188W); the immunofluorescence (HPA) and AP-MS data (BioPlex 2.0) on which the map is based; and data for the AP-MS pull-down experiments performed as follow-up. The new AP-MS data have also been included as part of the larger compendium of protein interactions in the next version of the BioPlex resource (BioPlex $3.0^{29}$ ). $\mathrm{AP}-\mathrm{MS}$ data, including filtered and unfiltered interaction lists as well as raw mass spectrometry data, are also available at http://bioplex. hms.harvard.edu. The image data and associated metadata can also be found in the HPA database (https://www.proteinatlas.org). The Gene Expression Omnibus (GEO) accession number for eCLIP data generated in this study is GSE171553. Source data are provided with this paper.

\section{Code availability}

The MuSIC pipeline is available at https://github.com/idekerlab/MuSIC along with a detailed step-by-step guide to building a MuSIC map.

31. Huang, G., Liu, Z., van der Maaten, L. \& Weinberger, K. Q. Densely connected convolutional networks. Preprint at https://arxiv.org/abs/1608.06993 (2016).

32. Nusinow, D. P. et al. Quantitative proteomics of the Cancer Cell Line Encyclopedia. Cell $180,387-402(2020)$

Acknowledgements We thank C. Ng, A. Palmer, Q. Zhang, Y. Quan, members of the laboratories of T.I. and E.L., the Human Protein Atlas and J. Swedlow for discussion and comments; M. Dow for helping us to improve the MuSIC GitHub repository and test the MuSIC pipeline; and the Cell Profiling facility and C. Stadler at the Science for Life Laboratory for help with in situ fractionation. This work was supported by the National Institutes of Health (NIH) under grants U54 CA209891, U01 MH115747, P41 GM103504 and R01 HG009979 to T.I. F99 CA264422 to Y.Q., U24 HG006673 to E.L.H., S.P.G. and J.W.H., U41 HG009889 and R01s HL137223 and HG004659 to G.W.Y. and R5O CA243885 to J.F.K.; by a gift from Google Ventures to J.W.H. and S.P.G.; by the Erling-Persson family foundation, Knut and Alice Wallenberg Foundation (2016.0204) and the Swedish Research Council (2017-05327) to E.L.; and by the Belgian Fonds de la Recherche Scientifique (F.R.S./FNRS), the Université Libre de Bruxelles (ULB), the European Joint Programme on Rare Diseases ('RiboEurope' and 'DBAcure'), the Région Wallonne (SPW EER) ('RIBOcancer'), the Internationale Brachet Stiftung and the Epitran COST action (CA16120) to D.L.J.L.

Author contributions Y.Q., E.L. and T.I. designed the study and developed the conceptual ideas. C.F.W. and W.O. generated image embeddings. Y.Q. and J.M. designed the data integration approach. Y.Q. and F.Z. designed the community detection approach. Y.Q., E.L.H., C.F.W., F.Z., L.V.S., W.O., J.P., A.P., J.F.K., J.M., J.W.H., E.L. and T.I. developed ideas for data analyses. Y.Q. implemented all computational methods and analyses. Y.Q., C.F.W., L.V.S., W.O. J.P. and T.I. organized the GitHub repository and wrote the step-by-step guide. Y.Q., E.L.H., C.F.W., M.R.K., L.P.V., E.S., J.F.K., S.P.G., J.W.H., G.W.Y., D.L.J.L., E.L. and T.I. designed validation experiments. E.L.H., L.P.V., T.Z., J.W.H. and S.P.G. generated and analysed AP-MS data and provided FLAG-HA-tagged clones. S.M.B. and G.W.Y. generated and analysed RIP-qPCR data. L.W. and D.L.J.L. generated and analysed northern blot data. C.F.W. A.B. and E.L. generated and analysed in situ fractionation data. M.L.G. and G.W.Y. generated and analysed eCLIP data. Y.Q., M.C., K.L. and J.J.L. performed the rest of the experiments. Y.Q., S.N.L. and T.I. designed the web portal page. Y.Q., E.L. and T.I. wrote the manuscript with input from all authors.

Competing interests T.I. is a co-founder of Data4Cure, is on the Scientific Advisory Board and has an equity interest. T.I. is on the Scientific Advisory Board of Ideaya BioSciences and has an equity interest. G.W.Y is a co-founder, a member of the Board of Directors, on the Scientific Advisory Board, an equity holder and a paid consultant for Locanabio and Eclipse

Biolnnovations. G.W.Y is a visiting professor at the National University of Singapore. The terms of these arrangements have been reviewed and approved by the University of California San Diego in accordance with its conflict-of-interest policies. E.L is on the Scientific Advisory Boards of Cartography Biosciences, Nautilus Biotechnology and Interline Therapeutics, and has an equity interest in all of these. J.W.H. is a co-founder of Caraway Therapeutics, is on the Scientific Advisory Board and has an equity interest. J.W.H. is Founding Scientific Advisor for Interline Therapeutics.

Additional information

Supplementary information The online version contains supplementary material available at https://doi.org/10.1038/s41586-021-04115-9.

Correspondence and requests for materials should be addressed to Emma Lundberg or Trey Ideker.

Peer review information Nature thanks Jason Swedlow and the other, anonymous, reviewer(s) for their contribution to the peer review of this work. Peer reviewer reports are available. Reprints and permissions information is available at http://www.nature.com/reprints. 

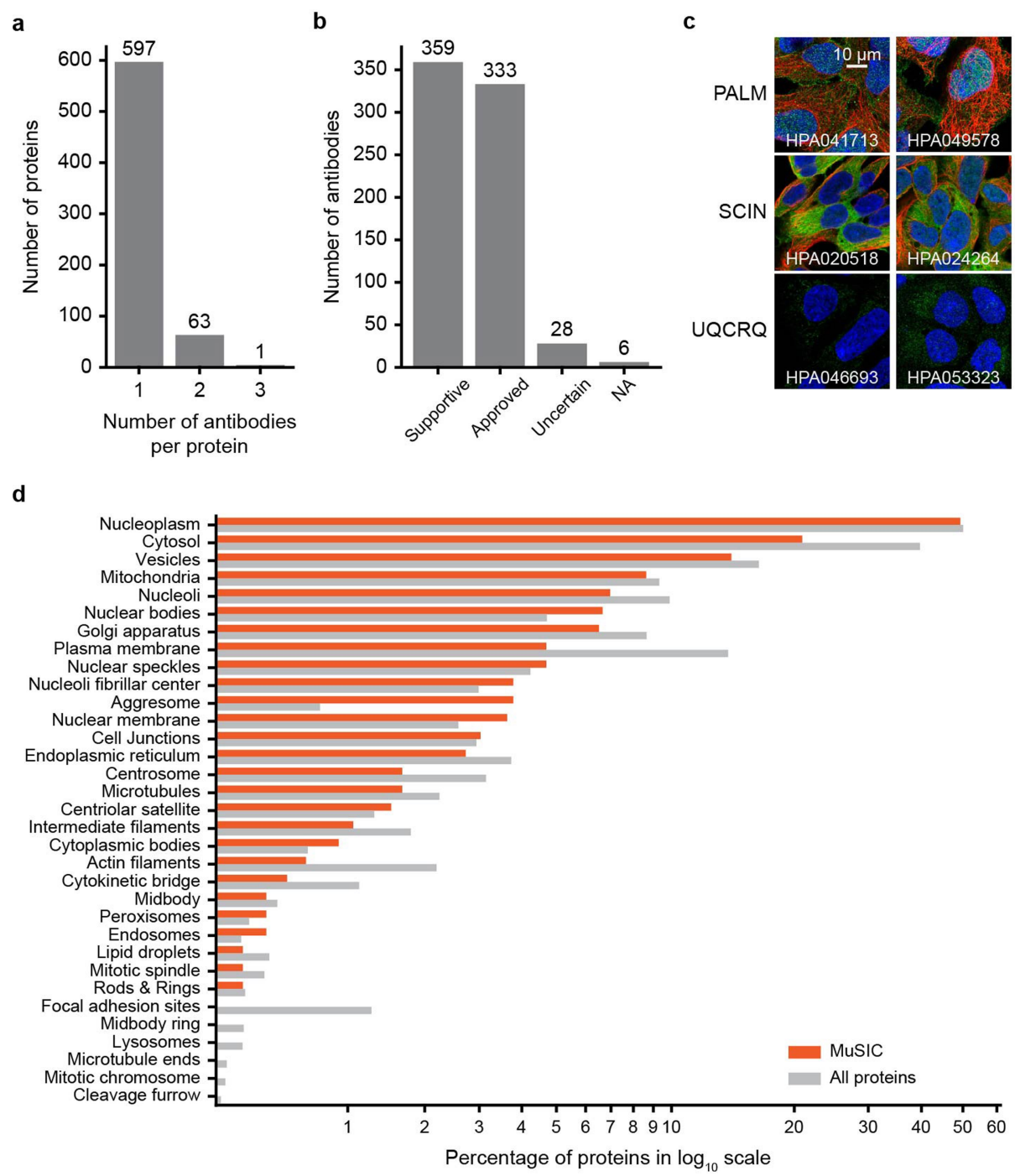

Extended Data Fig. 1 |Characterization of image data used in this study. a, Histogram showing distribution in number of antibodies per protein over 661 proteins included in MuSIC. b, Histogram showing distribution in antibody quality scores over antibodies used in this study. c, Immunofluorescence images for alternative antibodies (columns) targeting the same protein (rows).
Colours represent immunostained protein (green), cytoskeleton (red), or nucleus (blue). Images show high reproducibility for different antibodies against the same protein. d, Comparison of localizations for proteins in MuSIC (HEK293 cells, red) versus all proteins assayed by HPA in any cell line (grey). Localizations as defined by the HPA project ${ }^{4}$. 
a

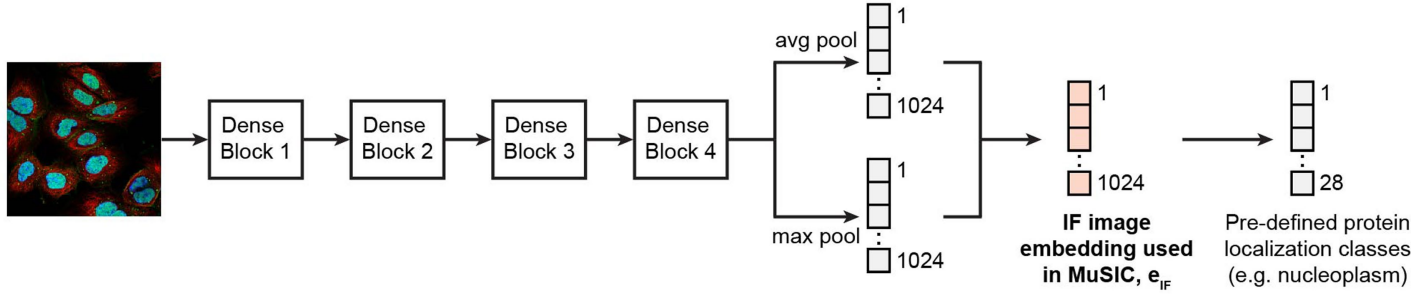

b

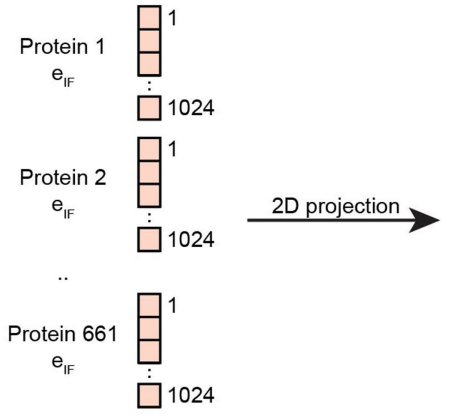

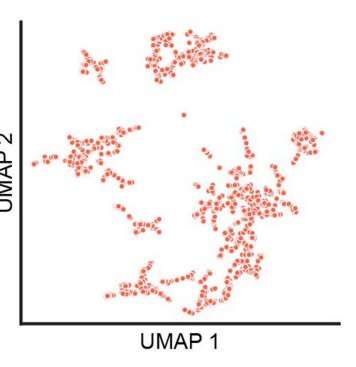

d

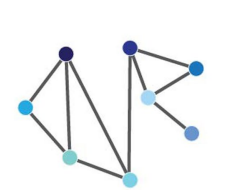

Physical protein association network constructed from AP-MS data

e

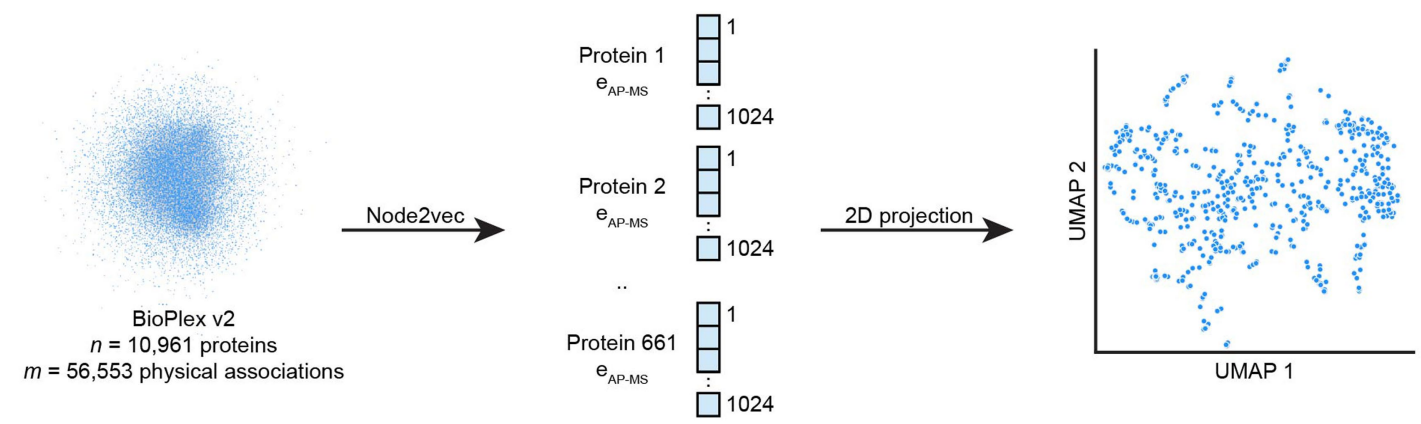

c

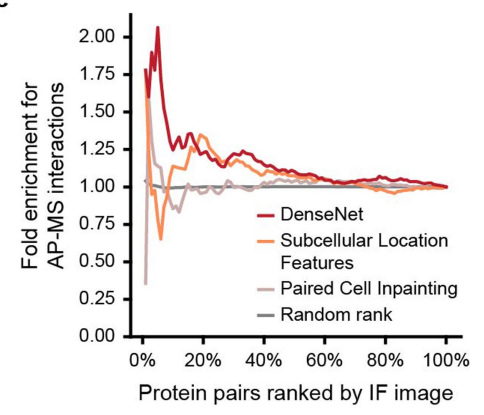

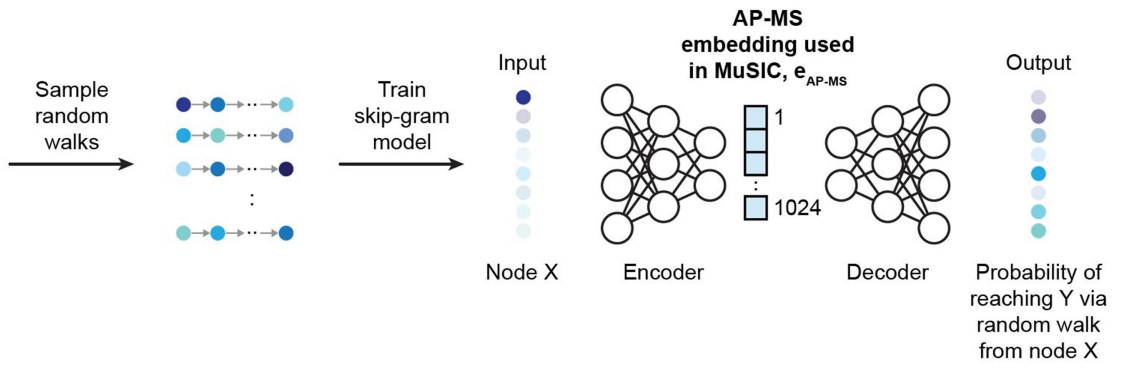

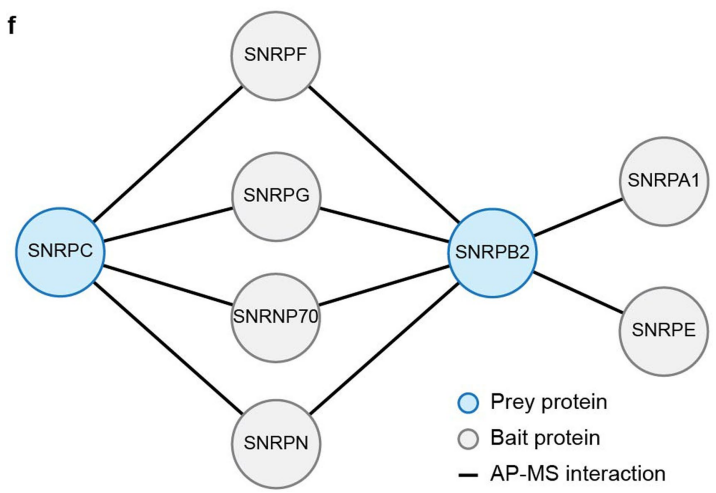

Extended Data Fig. 2 |See next page for caption. 


\section{Article}

Extended Data Fig. 2 |Embedding immunofluorescence images and AP-MS data. a, Embedding immunofluorescence (IF) images using DenseNet. The 1024-dimension feature vector for each IF image was extracted from a DenseNet-121 ${ }^{31}$ model trained to classify the IF image into one or several of 28 pre-defined protein localization classes from HPA.b. Two-dimensional visualization (UMAP, $n$ neighbours $=5$ ) for the 1,451 image embeddings associated with the 661 proteins in MuSIC.c, Ability of different image embedding methods (coloured curves) to generate image-image similarities (cosine similarity) in agreement with protein-protein interactions in BioPlex 2.0. d, Node $2 \mathrm{vec}^{8}$ workflow. The feature vector generated by node2vec captures the pattern of interaction neighbourhood for the respective node in input network. e, Embedding AP-MS data using node2vec. The input network to node $2 \mathrm{vec}$ was constructed by treating each protein as a node and assigning edges between protein pairs that were identified as physically interacting in the AP-MS data. The two-dimensional visualization (UMAP, $\mathrm{n}_{-}$neighbours =5) for AP-MS embeddings associated with 661 proteins in MuSIC is shown at right. f, Network showing all proteins (grey) that physically interact with SNRPC and SNRPB2 (blue) in BioPlex 2.0. SNRPC and SNRPB2 do not physically interact, but the cosine similarity of their embedded features is 0.93 due to shared interaction neighbourhood. In many cases of two proteins with high node2vec similarity but without direct interaction in AP-MS data, we found that neither protein had yet been tagged as bait for an affinity purification experiment. In these cases, the node2vec embedding suggests gaps in existing AP-MS data. g, Ability of different AP-MS embedding methods to generate protein-protein similarities (cosine similarity) in agreement with protein pairwise similarities computed from HPA images. 


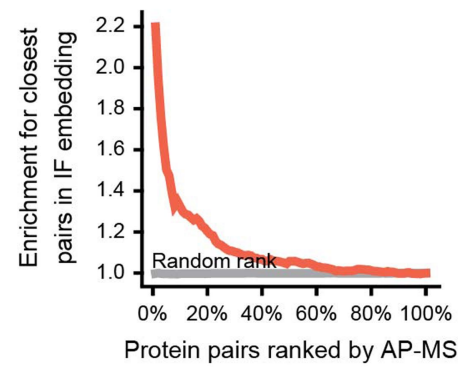

b
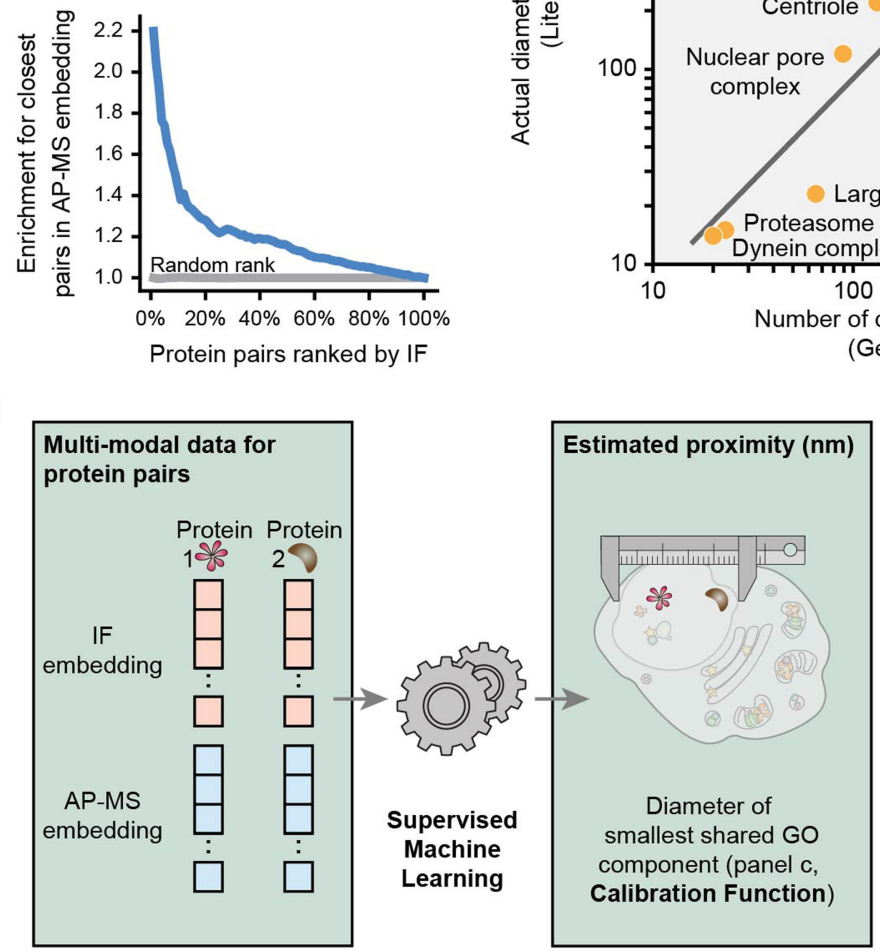

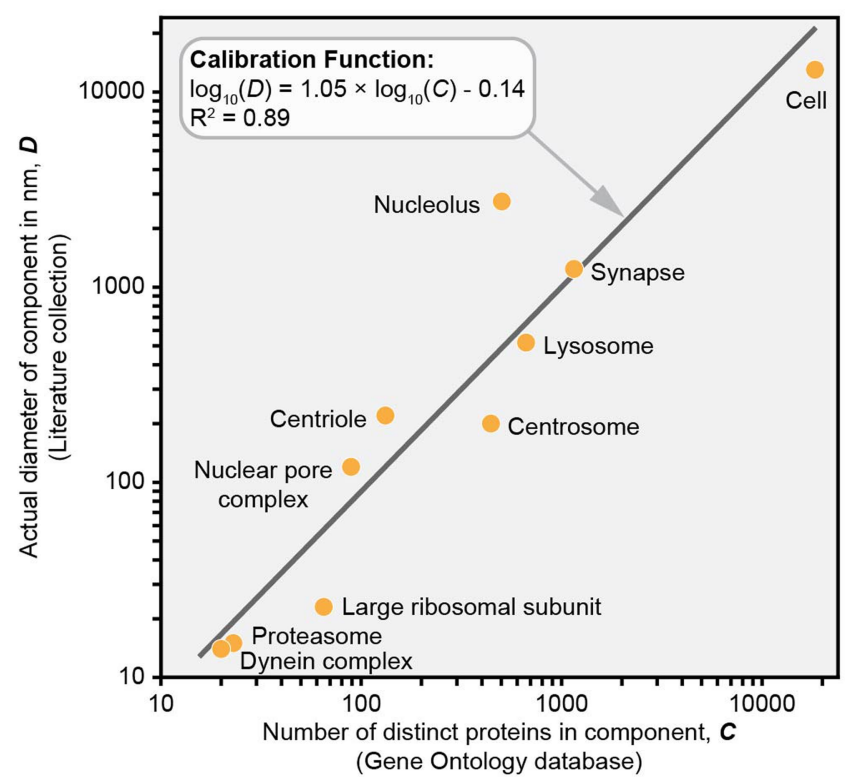
(Gene Ontology database)

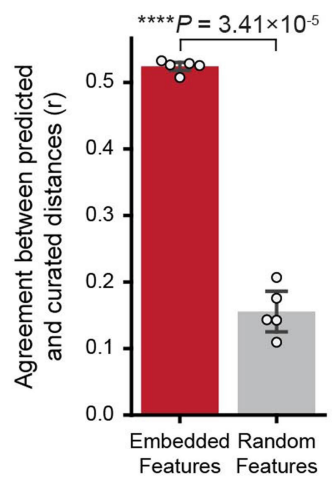

Extended Data Fig. 3 | Fusing protein distances from immunofluorescence and affinity purification. a, b, Protein pairs ranked by similarity in AP-MS embedding enrich for the most similar protein pairs in IF (a), and vice versa (b). c, Calibrating physical diameter, $D$, of subcellular components against the number of proteins, $C$, assigned to the corresponding Gene Ontology (GO) terms. d, Supervised model (random forest) estimates physical proximity $(\mathrm{nm})$ of all pairs of proteins from their IF and AP-MS embeddings. e, Performance of model in recovering protein-protein distances in $\mathrm{GO}$ in five-fold cross validation (red, Pearson's $r$ ). Equivalent calculation for random feature sets (grey). Statistics calculated using two-sided paired t-test. Data are presented as mean values $+/$ - standard deviation. 


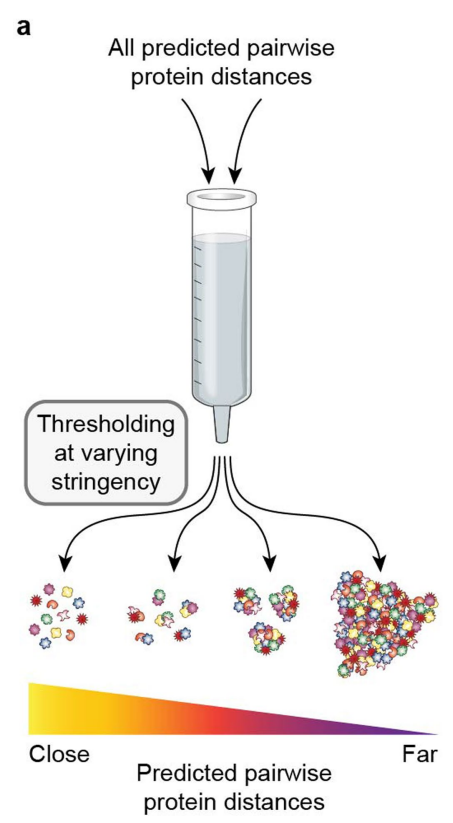

b

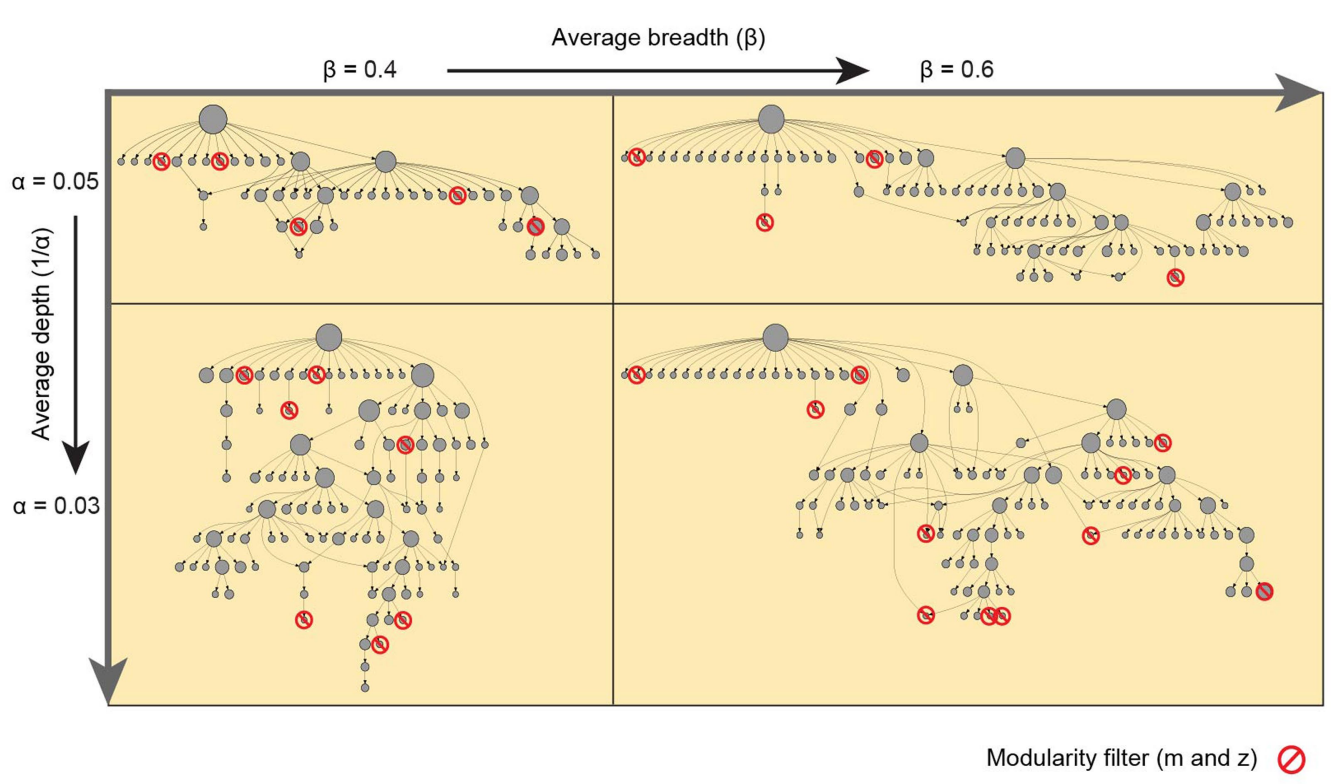

C

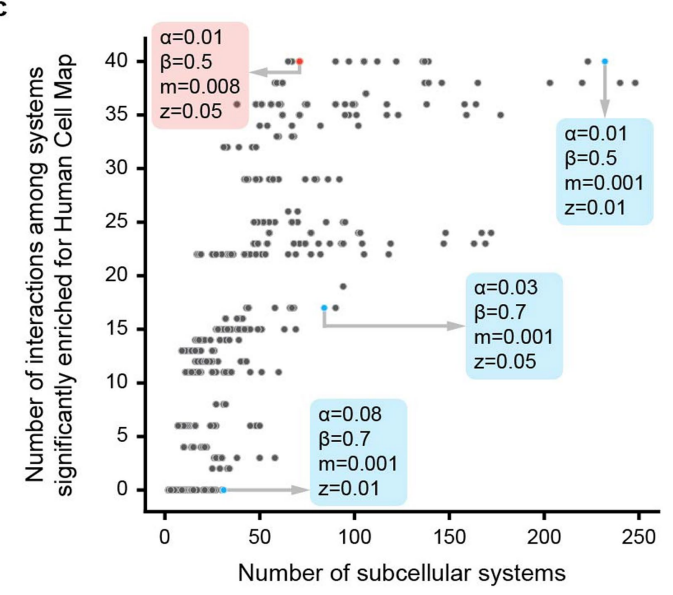

e

d
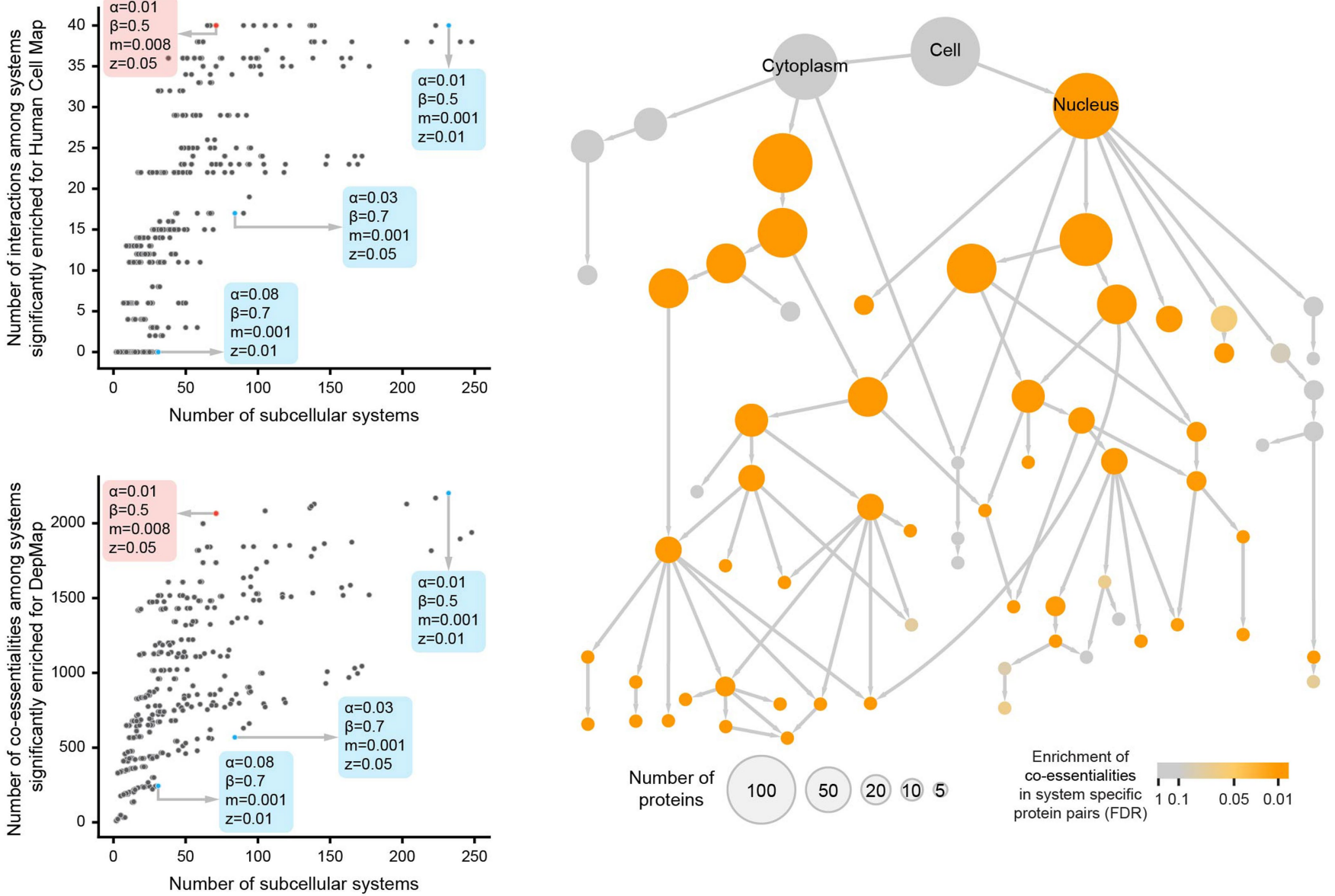

Extended Data Fig. 4 | Selection of parameters for community detection. a, Using multi-scale community detection, protein systems of increasing sizes are discovered as the threshold for protein-protein distance is progressively increased. b, CliXO community detection has four parameters (depth $\alpha, y$-axis; breadth $\beta, x$-axis; minimum modularity $m$ and modularity significance $z$, red circle backslash) that affect the sensitivity with which communities are identified and thus the size of the hierarchy. $\mathbf{c}, \mathbf{d}$, Dot plots in which each dot is a community hierarchy generated with a particular set of parameters. The

selection for MuSIC is highlighted in red. This selection was among several that were optimal based on enrichment for protein-protein interactions in Human Cell Map (c) and co-essentialities from DepMap (d). Examples of other parameter sets are shown in blue. e, Map from Fig. 2 with system colour showing enrichment for co-essentialities among protein pairs that are specific to that system. Enrichment of each system is assessed empirically, using 1,000 randomized hierarchies, followed by Benjamini-Hochberg multiple test correction to obtain FDR (orange gradient). 
a
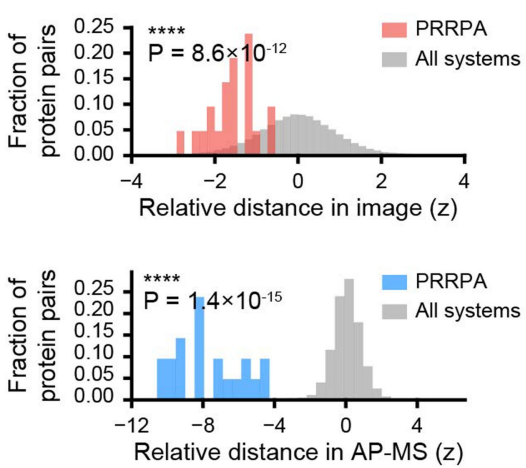

d

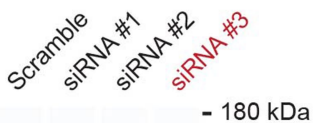

KRI1

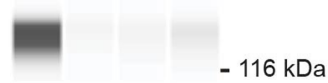

GAPDH

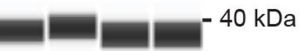

g

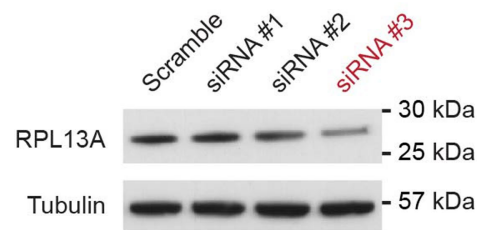

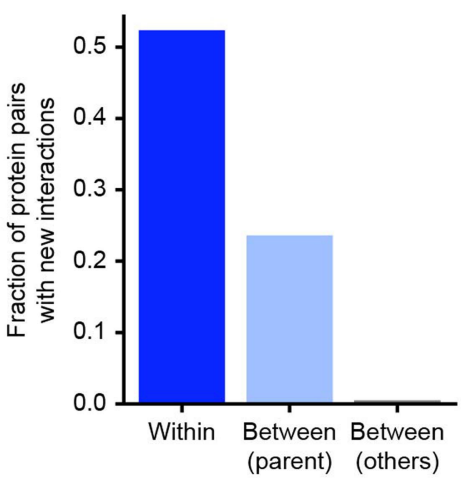

e

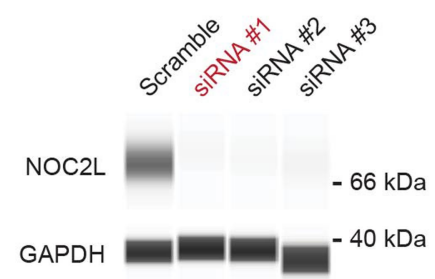

h

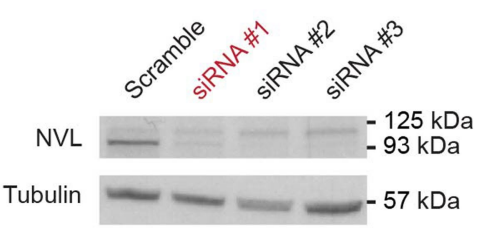

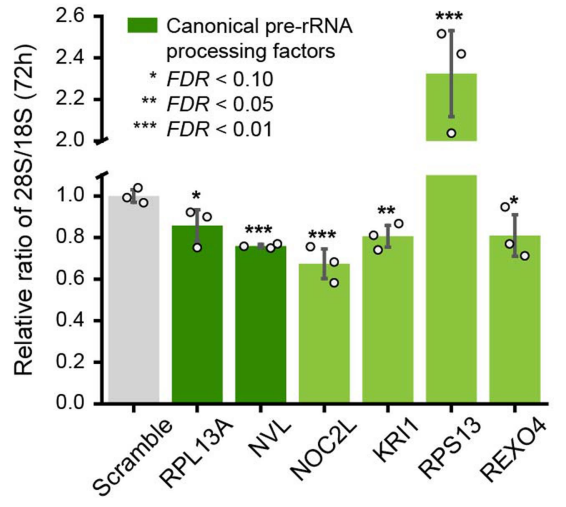

f
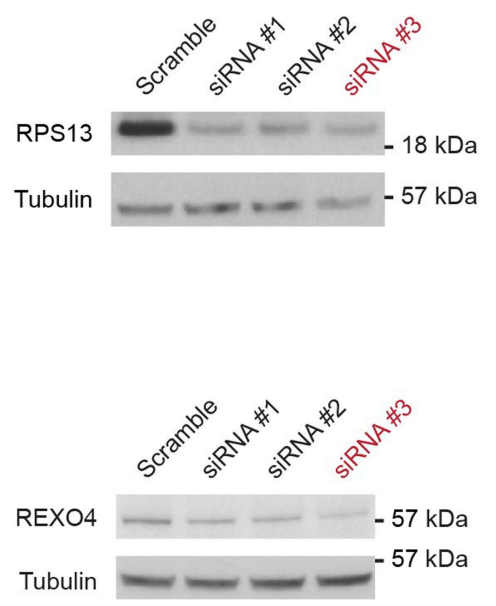

Extended Data Fig. 5 |Supporting analyses for PRRPA. a, Distributions of protein-protein distance $z$-scores among the seven proteins in the PRRPA system for IF (top, red) or AP-MS (bottom, blue) modalities, calibrated to all such distances, respectively (grey). Statistics calculated using one-sided Mann-Whitney $U$ test. b, Specific recovery of new AP-MS interactions within PRRPA is shown (dark blue bar), in comparison to interactions between proteins in PRRPA and other proteins organized under the same parent systems ("Ribosome" and "Ribosome biogenesis assembly", light blue bar), or between proteins in PRRPA and those organized elsewhere in MuSIC (grey bar). c, Mature 28S/18S rRNA ratio under siRNAs targeting each PRRPA protein (green) versus scrambled siRNA (grey), $n=3$ biological replicates. FDR from two-sided t-test with Benjamini-Hochberg correction. Data are presented as mean values +/- standard deviation. d-i, Western blot analysis (d,e, Simple western assay; $\mathbf{f}-\mathbf{i}$, SDS-PAGE) of target protein abundance after treating HEK293T cells with respective siRNA for $72 \mathrm{~h}$ (Supplementary Tables 6, 7). The siRNAs highlighted in red were selected to assess the perturbation of mature rRNA ratio (28S/18S rRNA) when knocking down target protein, with protein knockdown efficiency confirmed using western blot in three additional biological replicates. For source data, see Supplementary Fig. 1 (gel; d-i) and Supplementary Fig. 2 (total RNA profiles; c). 


\section{Article}

a

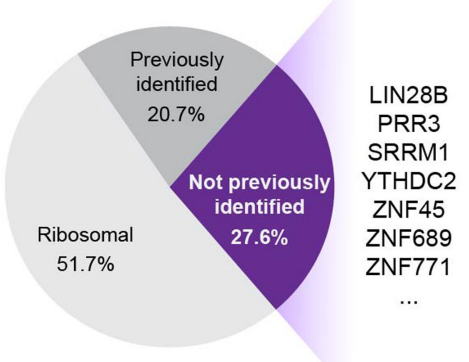

d

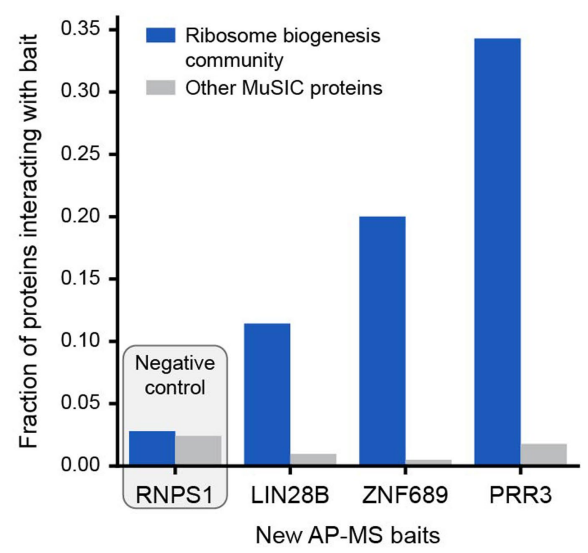

e
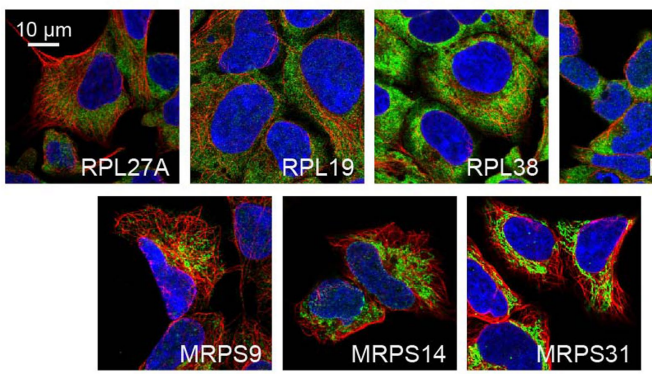

b

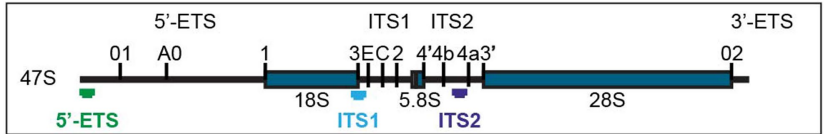

c
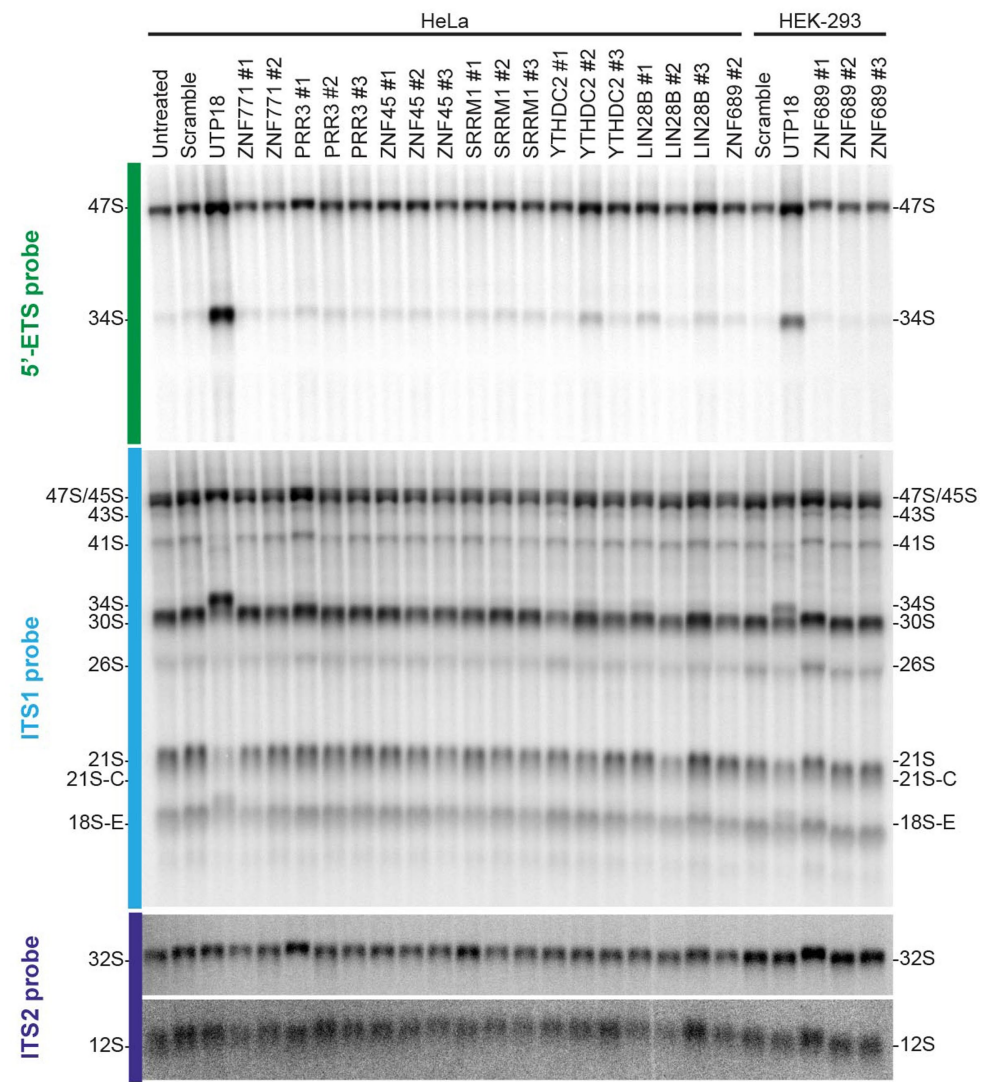

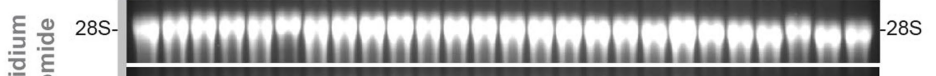

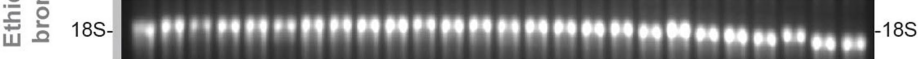
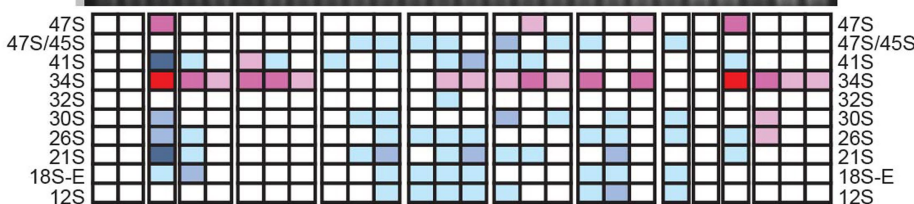

$<25 \% \square 25-50 \% \quad \square 50-67 \% \square 67-125 \% \square 125-175 \% \square 175-400 \% \square>400 \%$ f

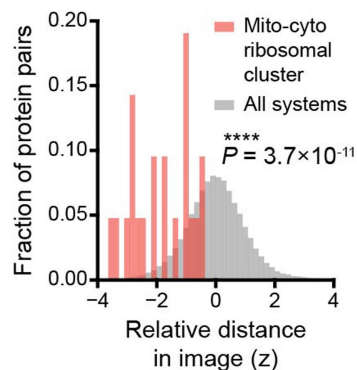

g

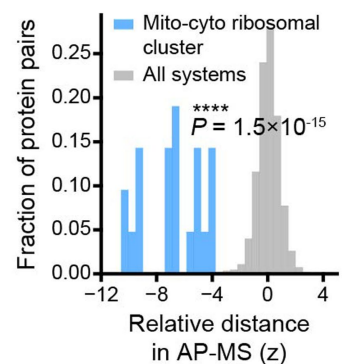

h

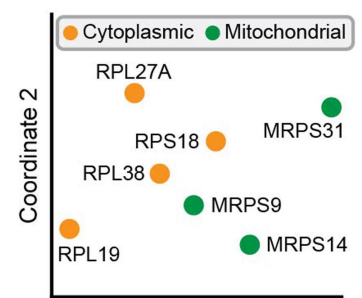

Coordinate 1 i

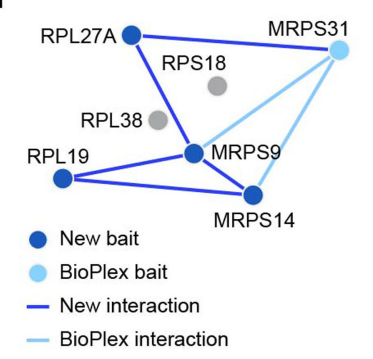

Extended Data Fig. 6 |See next page for caption. 
Extended Data Fig. 6 | Supporting analyses for ribosomal systems.

a, Categorization of proteins in "Ribosome biogenesis community" by whether they have been previously identified in human ribosome biogenesis. Excludes PRRPA proteins described in Fig. $5 \mathbf{b}-\mathrm{d}$. $\mathbf{b}$, Structure of human pre-rRNA and probes used for northern blot. In eukaryotes, 3 out of 4 mature rRNAs (18S, $5.8 \mathrm{~S}$, and $28 \mathrm{~S}$ rRNAs) are produced from a single long polycistronic precursor (47S) synthesized by RNA polymerase I. The mature rRNAs are interspersed with the $5^{\prime}$ and $3^{\prime}$ external transcribed spacers (ETS) and internal transcribed spacer (ITS) 1 and 2 . The probes used in the northern blot (5'-ETS, ITS1, and ITS2) are indicated and colour-coded.c, Total RNA extracted from the indicated cell line, which was transfected with a DsiRNA specific to the target protein for $72 \mathrm{~h}$ and analysed by northern blotting with probes specific to the 5'-ETS, ITS1, and ITS2 sequences (Supplementary Table 8). As controls, cells were either untreated, transfected with a scrambled silencer, or transfected with a silencer targeting UTP18 (positive control involved in small ribosomal subunit biogenesis). Heat map colour shows the percentage of each pre-rRNA species with respect to the scramble control. For gel source data, see Supplementary Fig. 1.d, For protein baits in new AP-MS experiments (x axis), fraction of interacting preys that fall within the Ribosome biogenesis community (blue bars) versus elsewhere (grey bars). Only new AP-MS interactions are considered for this analysis. RNPS1 does not belong to Ribosome biogenesis community and serves as a negative control.e, IF images showing similar cytoplasmic staining for proteins in “Mito-cyto ribosomal cluster." Cytoplasmic staining is dim for MRPS9, MRPS14 and MRPS31 compared to their predominant mitochondrial locations. Colours represent immunostained protein (green), cytoskeleton (red) and nucleus (blue). $\mathbf{f}, \mathbf{g}$, Corresponding distributions of protein-protein distance z-scores for IF (f, red) or AP-MS (g, blue), calibrated to all such distances, respectively (grey). Statistics calculated using one-sided Mann-Whitney $U$ test. h, Twodimensional projection of proteins in Mito-cyto ribosomal cluster, as in Fig. $5 f$. Proteins coloured according to known affiliations to cytoplasmic ribosome or mitochondrial ribosome. i, Validated AP-MS interactions in Mito-cyto ribosomal cluster. Note that only one out of seven proteins was previously tagged as bait in BioPlex 2.0 (light blue node), thus most physical associations (dark blue edges) among protein pairs were newly identified in this study. 

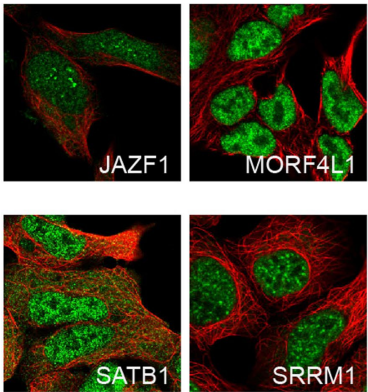

d
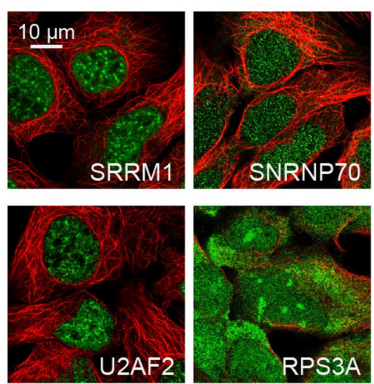

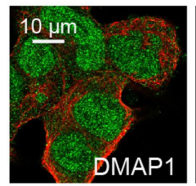

b
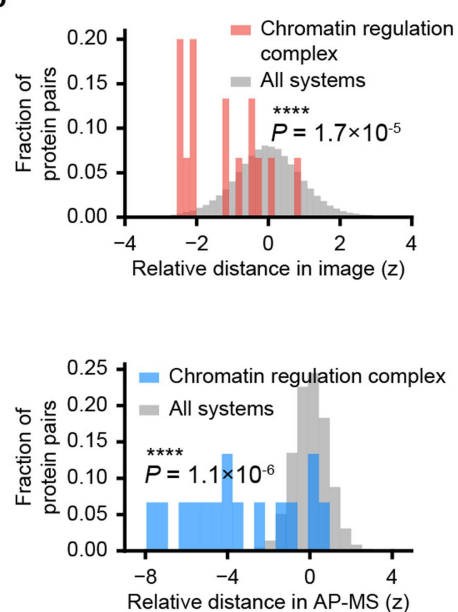

e
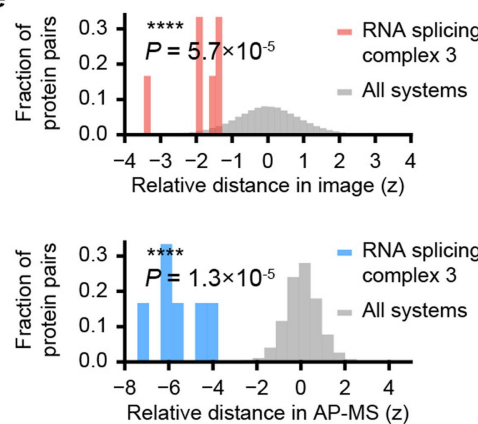

h c
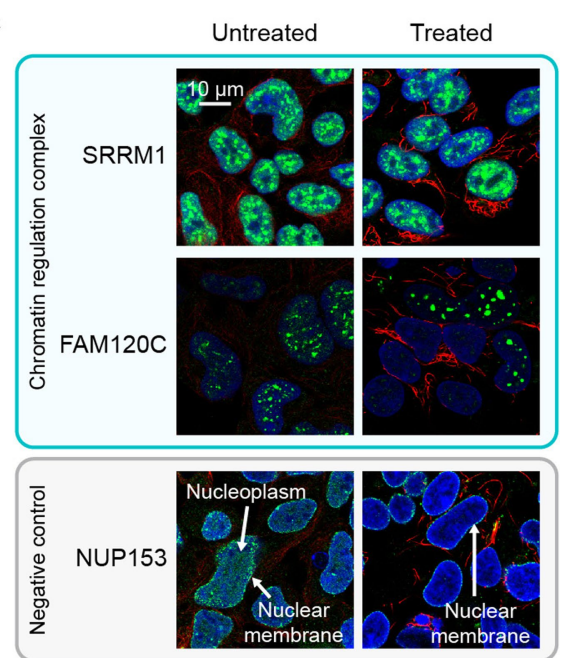

f

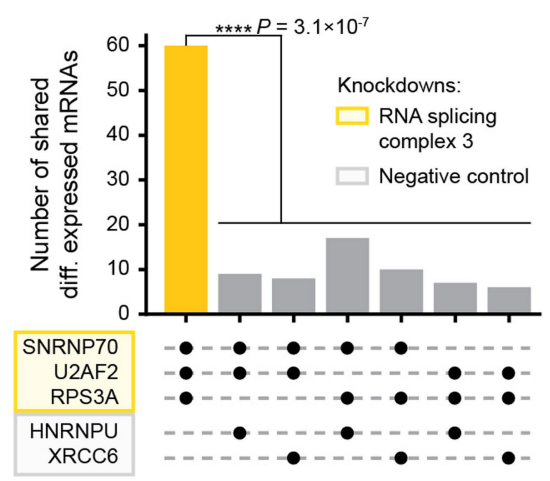

g
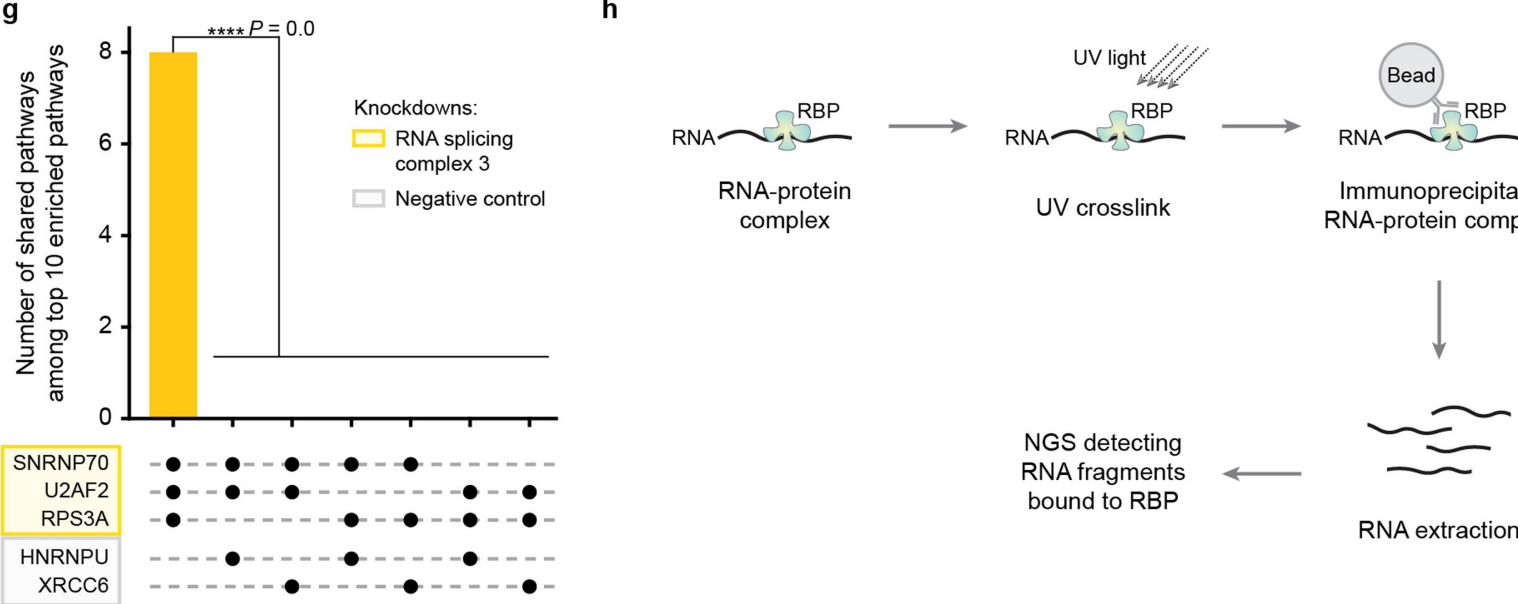

UV crosslink

Immunoprecipitate RNA-protein complex

NGS detecting

RNA fragments

bound to RBP

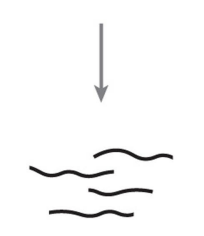

RNA extraction
Extended Data Fig. 7 |Supporting analyses for chromatin regulation and splicing systems. a, IF images showing similar nucleoplasm and nuclear speckles signals among proteins in the "Chromatin regulation complex." Colours represent immunostained protein (green) and cytoskeleton (red). b, Distributions of pairwise protein distance $z$-scores among the proteins in the Chromatin regulation complex for IF (top, red) or AP-MS (bottom, blue) modalities, calibrated to all such distances, respectively (grey). Statistics calculated using one-sided Mann-Whitney $U$ test. c, Immunofluorescent proteins (rows) imaged in HEK293 cells, untreated (left) or treated (right) with in situ fractionation to remove soluble cytoplasmic and loosely held nuclear proteins. Chromatin-binding proteins remain after treatment. Blue, nucleus; other colours as in a. For image source data, see Supplementary Fig. 3. d, IF images showing similar nucleoplasm signals among proteins in "RNA splicing complex 3." e, Similar display for RNA splicing complex 3 as in b.f, Comparison of 500 top differentially expressed mRNAs (absolute fold change) resulting from shRNA knockdown of each of five genes (see Supplementary Table 9 for file accessions). Bar chart shows number of differential mRNAs shared by different gene groups indicated by black dots beneath each bar. One-sided one-sample t-test. g, Comparison among the top 10 pathways (Gene Ontology Biological Process) returned from Gene Set Enrichment Analysis using the top 500 differentially expressed transcripts. Bar chart shows number of enriched pathways shared by different gene groups indicated by black dots beneath each bar. One-sided one-sample t-test. h, eCLIP workflow. RBP, RNA-binding protein. NGS, next generation sequencing. 
a
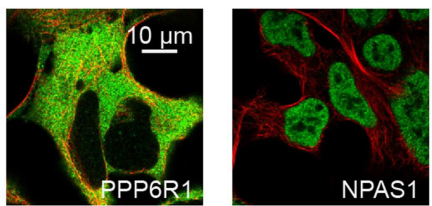

b

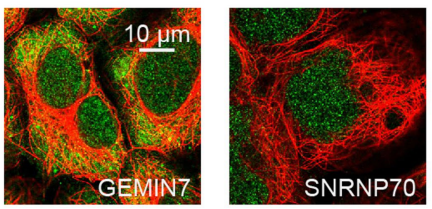

C

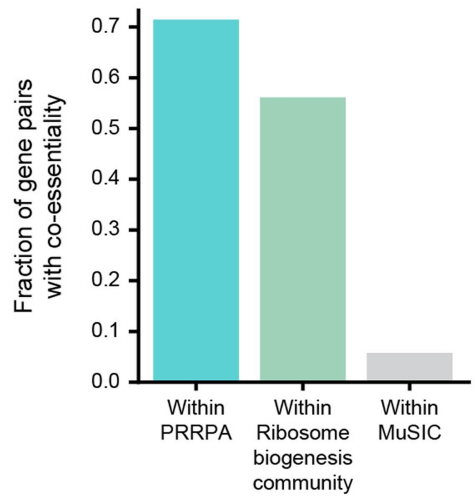

d

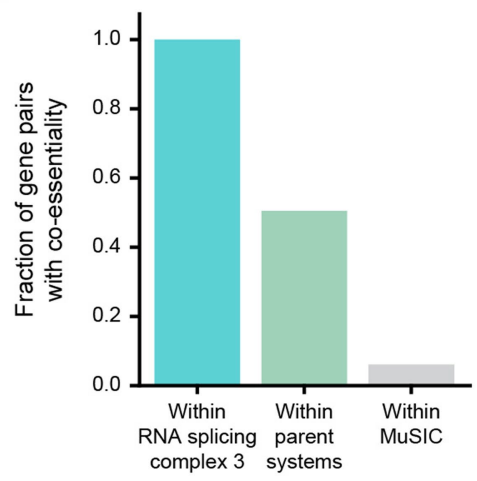

e

Co

Large ribosomal subunit subcomplex 4

Large ribosomal subunit subcomplex 3 -

Cotranslational protein targeting to membrane system -

Ribosomal complex 1

Ribosomal complex 5

Ribosomal complex 2 -

Ribosomal complex 3 -

RNA splicing complex 2 -

Splicing regulatory complex -

U2-type spliceosomal complex family

Ribosomal complex 4

Subgroup of spliceosomal complex family

Spliceosomal complex family -

Catalytic step 2 spliceosome

Large ribosomal subunit subcomplex 2 -

U2 snRNP
U1 snRNP
PRRPA

Pre-catalytic spliceosome

RNA splicing complex 1 Mitochondrion

Small nuclear ribonucleoprotein complex family -

Pre-catalytic spliceosome subcomplex 1

Chromatin regulation assembly -

Mito-cyto ribosomal cluster

NuA4 histone acetyltransferase complex -

RNA splicing complex 3

Polysomal ribosome complex -

Pre-mRNA splicing complex 1

Mitochondrial large ribosomal subunit

Histone acetyltransferase complex family

DNA metabolic assembly

Ribosome biogenesis subcomplex

Chromosome organization complex family

Large ribosomal subunit subcomplex 1 Nucleoplasm 1

Chromatin organization complex family Chromatin regulation complex

Nuclear transcriptional speckle

Nucleic acid binding complex

Negative regulation of RNA biosynthetic process Ribosomal complex 6 -

RNA processing complex 1 -

RNA processing complex 2

$$
\text { L }
$$

FDR

$$
[0,0.01]
$$

$(0.01,0.05]$

$(0.05,0.1]$

- Non-significant

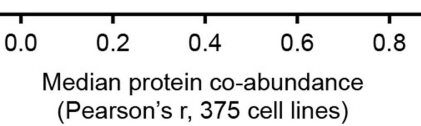

(Pearson's r, 375 cell lines)
Extended Data Fig. 8 | Supporting analyses for Discussion. a, b, Examples of proteins with strong AP-MS protein interactions that have very different IF localization patterns. Colours represent immunostained protein (green) and cytoskeleton (red). c, Degree of co-essentiality for gene pairs within PRRPA (teal bar) shown in comparison to remaining pairs of genes assigned to the more general system that contains it, "Ribosome biogenesis community" (green bar), as well as all other gene pairs in MuSIC (grey bar). d, Similar analysis as in (c) for "RNA splicing complex 3." Parent systems are "RNA processing complex 1" and "RNA splicing complex family." e, Protein co-abundance for MuSIC systems, calculated from the median Pearson correlation of pairwise protein abundance over 375 diverse cell lines ${ }^{32}$. The plot shows all systems with fewer than 20 proteins and co-abundance measurements for $>50 \%$ of protein pairs. Significance is assessed empirically (one-sided), using 1,000 randomized MuSIC hierarchies, followed by Benjamini-Hochberg multiple test correction to obtain FDR (colour of bar). Protein co-abundance for a system provides evidence for its presence in cell types beyond HEK 293. 


\section{Reporting Summary}

Nature Research wishes to improve the reproducibility of the work that we publish. This form provides structure for consistency and transparency in reporting. For further information on Nature Research policies, see our Editorial Policies and the Editorial Policy Checklist.

\section{Statistics}

For all statistical analyses, confirm that the following items are present in the figure legend, table legend, main text, or Methods section.

n/a Confirmed

$\bigotimes$ The exact sample size $(n)$ for each experimental group/condition, given as a discrete number and unit of measurement

$\square$ \ A statement on whether measurements were taken from distinct samples or whether the same sample was measured repeatedly

$\square$ The statistical test(s) used AND whether they are one- or two-sided

$\square$ Only common tests should be described solely by name; describe more complex techniques in the Methods section.

Х $\square$ A description of all covariates tested

$\square$ \A description of any assumptions or corrections, such as tests of normality and adjustment for multiple comparisons

$\square$ A full description of the statistical parameters including central tendency (e.g. means) or other basic estimates (e.g. regression coefficient) AND variation (e.g. standard deviation) or associated estimates of uncertainty (e.g. confidence intervals)

$\varnothing$ For null hypothesis testing, the test statistic (e.g. $F, t, r$ ) with confidence intervals, effect sizes, degrees of freedom and $P$ value noted

Give P values as exact values whenever suitable.

Х $\square$ For Bayesian analysis, information on the choice of priors and Markov chain Monte Carlo settings

$\square$ \ For hierarchical and complex designs, identification of the appropriate level for tests and full reporting of outcomes

$\square$ Estimates of effect sizes (e.g. Cohen's $d$, Pearson's $r$ ), indicating how they were calculated

Our web collection on statistics for biologists contains articles on many of the points above.

\section{Software and code}

Policy information about availability of computer code

Data collection Immunofluorescence confocal images were collected using 63x oil immersion with Numerical Aperture 1.4. AP-MS data were acquired on first-generation Q-Exactive mass spectrometers (Thermo Fisher Scientific) equipped with Famos autosamplers (LC Packings) and Accela600 liquid chromatography (LC) pumps (Thermo Fisher Scientific). The eCLIP data were sequenced using Illumina HiSeq 4000.

Data analysis All data analyses have been described in detail in the relevant Methods section with links to publicly available GitHub repositories. The MuSIC pipeline, along with a detailed step-by-step guide to reproduce MuSIC 1.0 presented in the paper, are included in a publicly available GitHub repository https://github.com/idekerlab/MuSIC. The required Python packages, along with specific versions, were documented and wrapped as an installation file in GitHub. These packages include:

dill==0.3.1.1

distributed $==1.18 .3$

docutils $==0.14$

entrypoints $==0.2 .3$

gensim $==4.0 .1$

glob2 $==0.5$

gmpy $2==2.0 .8$

imageio $==2.2 .0$

imagesize $==0.7 .1$

imutils $==0.5 .3$

loess $==2.0 .11$

louvain==0.6.1

matplotlib $>=2.0 .2$

mistune $==0.7 .4$

nbconvert $==5.3 .1$

nbformat $==4.4 .0$ 


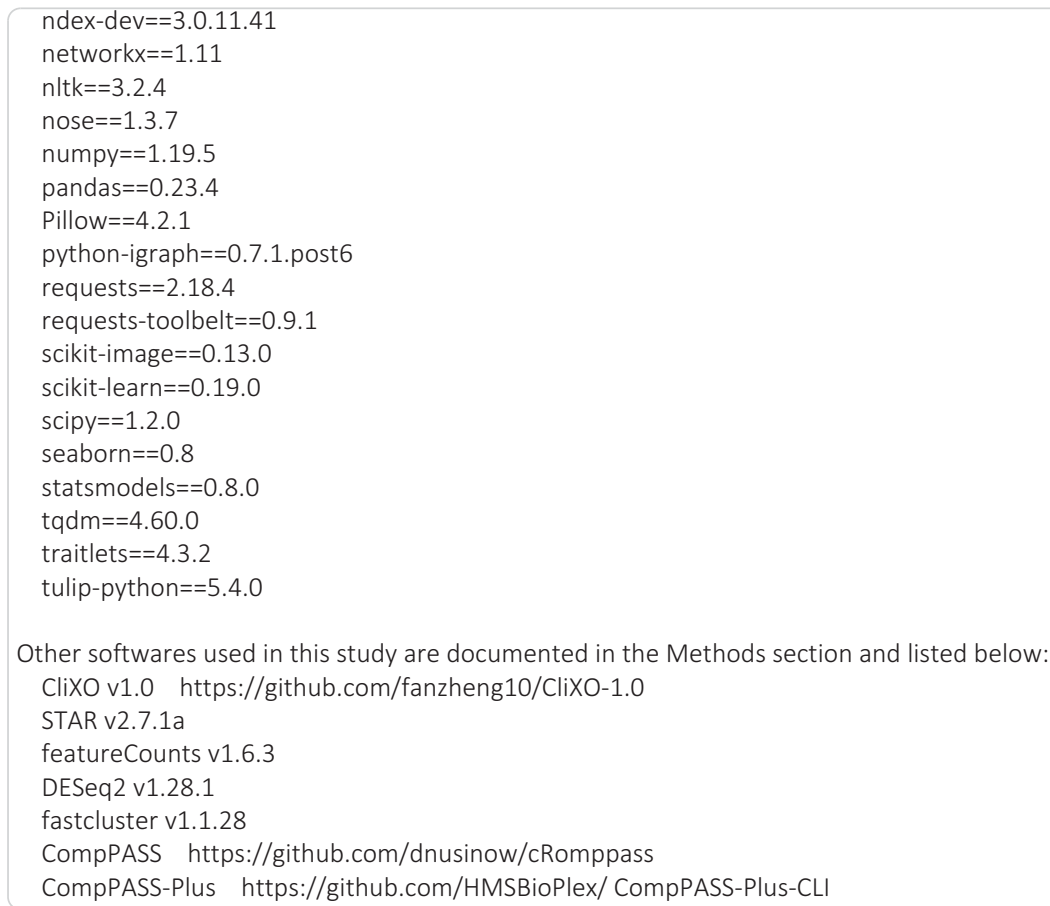

Other softwares used in this study are documented in the Methods section and listed below:

CliXO v1.0 https://github.com/fanzheng10/CliXO-1.0

STAR V2.7.1a

featureCounts v1.6.3

DESeq2 v1.28.1

fastcluster v1.1.28

CompPASS https://github.com/dnusinow/cRomppass

CompPASS-Plus https://github.com/HMSBioPlex/CompPASS-Plus-CLI

For manuscripts utilizing custom algorithms or software that are central to the research but not yet described in published literature, software must be made available to editors and reviewers. We strongly encourage code deposition in a community repository (e.g. GitHub). See the Nature Research guidelines for submitting code \& software for further information.

\section{Data}

Policy information about availability of data

All manuscripts must include a data availability statement. This statement should provide the following information, where applicable:

- Accession codes, unique identifiers, or web links for publicly available datasets

- A list of figures that have associated raw data

- A description of any restrictions on data availability

A web portal is available at http://nrnb.org/music with links to all major resources used for this study. These include the MuSIC systems map; the immunofluorescence (HPA) and AP-MS data (BioPlex 2.0) on which the map is based; and data for the AP-MS pulldown experiments performed as follow-up. The new AP-MS data have also been included as part of the larger compendium of protein interactions to be included in the next version of the BioPlex resource (BioPlex 3.0). AP-MS data, including filtered and unfiltered interaction lists as well as raw mass spectrometry data, are also available at http:// bioplex.hms.harvard.edu. The Gene Expression Omnibus (GEO) accession number for eCLIP data generated in this study is GSE171553.

Other public databases used in this study are documented in the Methods section and listed below:

Gene Ontology (http://geneontology.org/; 25.9.2018 release)

ENCODE (https://www.encodeproject.org/; v3)

Human Cell Map (https://cell-map.org/; v1)

Cancer Cell Dependency Map (https://depmap.org/portal/; v20Q3)

Human ORFeome, version 8.1

UniProt database (includes both SwissProt and Trembl entries and dates to the outset of the BioPlex study in 2013)

\section{Field-specific reporting}

Please select the one below that is the best fit for your research. If you are not sure, read the appropriate sections before making your selection. \Life sciences Behavioural \& social sciences Ecological, evolutionary \& environmental sciences

For a reference copy of the document with all sections, see nature.com/documents/nr-reporting-summary-flat.pdf

\section{Life sciences study design}

All studies must disclose on these points even when the disclosure is negative.

Sample size

The number of proteins analyzed in this study ( $n=661)$ was determined based on amount of matched data available when overlapping Human Protein Atlas (HPA) and BioPlex. Other proteins in HPA and BioPlex were measured in differing cell types that did not align across projects; thus, we focused on the 661 proteins in the common HEK293-derived context for prototyping the new approach we developed in this study. As we have demonstrated in Extended Data Fig. 1d, these proteins covered a wide distribution of subcellular locations, as previously annotated by HPA, which was very similar to the distribution seen for all human proteins. 
For all follow-up experiments in this study, no statistical methods were used to pre-determine sample sizes. The sample sizes were chosen to reliably observe experimental phenotypes.

Data exclusions RPL6 was excluded from downstream analysis of MuSIC map due to concerns for antibody correctness in IF staining. For 28S/18S measurement shown in Extended Data Fig. 5c, RPL7L1 was excluded due to technical difficulties. For RIP-qPCR data shown in Fig. 5d, RPL13A was excluded due to technical difficulties.

Replication All the data collected in this study consisted of technical or biological replicates. The number of replicates, as well as the type of replicates (i.e. technical or biological), are labeled in the relevant figures or method sections.

Randomization AP-MS baits were arrayed on 96-well plates in random order, and plates were run in random order during LC-MS analysis. For other experiments in this study, randomization was used whenever possible to determine experimental order, but individual samples were not intentionally randomized since this does not affect the results.

Blinding All AP-MS data and eCLIP data were generated and processed with investigators blinded to the hypothesis.

For the RIP-qPCR data (Fig. 5d), the experimenter was blinded to the group allocation. Blinding was not applied during analysis, which we followed established procedure from previous studies and provided related source data.

For the northern blot data (Fig. 5e), the investigators were blinded to group allocation during data acquisition and analysis. Related source data are provided as well.

For 28S/18S measurement (Extended Data Fig. 5c), the investigator was not blinded to the group allocation. We designed stringent computational pipeline that algorithmically fit Gaussian curves to analyze the 18S and 28S rRNA abundance from TapeStation profiles (Supplementary Figure 2) and provided related source data.

For in situ fractionation data (Extended Data Fig. 7c), the experimenter was blinded to the group allocation. Blinding was not applied during analysis. Related source data are provided in Supplementary Figure 3.

\section{Reporting for specific materials, systems and methods}

We require information from authors about some types of materials, experimental systems and methods used in many studies. Here, indicate whether each material, system or method listed is relevant to your study. If you are not sure if a list item applies to your research, read the appropriate section before selecting a response.

\begin{tabular}{|c|c|}
\hline$n / a$ & Involved in the study \\
\hline & Х Antibodies \\
\hline & \ Eukaryotic cell lines \\
\hline$\bigotimes$ & Palaeontology and archaeology \\
\hline$\bigotimes$ & Animals and other organisms \\
\hline$\bigotimes$ & Human research participants \\
\hline Х & Clinical data \\
\hline$\bigotimes$ & Dual use research of concern \\
\hline
\end{tabular}

\begin{tabular}{l|l}
\multicolumn{2}{l}{ Methods } \\
\hline n/a & Involved in the study \\
$\bigotimes$ & $\square$ ChIP-seq \\
$\bigotimes$ & $\square$ Flow cytometry \\
$\bigotimes$ & $\square$ MRI-based neuroimaging
\end{tabular}

\section{Antibodies}

Antibodies used

Validation
All the antibodies used in this study, including respective catalog number and dilution used, are provided in Supplementary Table 7 and reproduced below:

GAPDH NBP1-47339 1:4000 (ProteinSimple WES)

HA-tag Sigma-Aldrich H9658; BioLegend \#901501; CST \#3724 Western blot - 1:10000; eCLIP - see protocol; AP-MS - see protocol KRI1 Novus NBP2-14797 $3 \mathrm{ug} / \mathrm{mL}$ (ProteinSimple WES)

Mouse secondary Cell Signaling Technology 70765 1:5000

NOC2L Novus NBP1-92190 $0.5 \mathrm{ug} / \mathrm{mL}$ (ProteinSimple WES)

NVL LS-C352601 1:500

Rabbit secondary Cell Signaling Technology 7074S 1:5000

Rat secondary Jackson ImmunoResearch 712-035-153 1:5000

REXO4 Novus NBP2-85628 1:500

RPL13A Novus NBP1-32710 1:500

RPS13 Novus NBP2-93953 1:500

Tubulin Abcam ab196583 1:10000

The primary antibodies used for validating protein knockdown in this study showed decreased protein abundance around expected molecular weight for samples treated with siRNA. Furthermore, all antibodies used in this study are commercially available and have been validated by the manufacturer, for which the evidences and relevant criteria are available in the following links:

Tubulin https://www.abcam.com/hrp-tubulin-antibody-yol134-microtubule-marker-ab196583.html

RPS13 https://www.novusbio.com/products/rps13-antibody_nbp2-93953

REXO4 https://www.novusbio.com/products/rexo4-antibody nbp2-85628

RPL13A https://www.novusbio.com/products/rpl13a-antibody_nbp1-32710

KRI1 https://www.novusbio.com/products/kri1-antibody_nbp2-14797

NVL https://www.Isbio.com/antibodies/nvl-antibody-internal-wb-western-ls-c352601/363722 
NOC2L https://www.novusbio.com/products/noc2l-antibody_nbp1-92190

HA-tag https://www.sigmaaldrich.com/catalog/product/sigma/h9658?lang=en\&region=US

https://www.biolegend.com/fr-ch/global-elements/pdf-popup/purified-anti-ha-11-epitope-tag-antibody-11374

https://www.cellsignal.com/products/primary-antibodies/ha-tag-c29f4-rabbit-mab/3724

GAPDH https://www.novusbio.com/products/gapdh-antibody-1a10_nbp1-47339

\section{Eukaryotic cell lines}

Policy information about cell lines

Cell line source(s)

HEK293T cells used for AP-MS were from American Type Culture Collection (ATCC). Cell lines used for pre-rRNA analysis (HeLa ref. ATCC CCL-2; HEK293 ref. ATCC CRL-1573) were purchased from ATCC. HEK293 cells used for IF stainings were obtained from ATCC.

Authentication

The identity of HEK293T cell line used for AP-MS experiments was verified through GTG-banded karyotyping by the Brigham and Women's Hospital Cytogenomics Core Laboratory.

The identity of other HEK293T cell lines used in this study was validated by the CellCheck service provided at IDEXX BioAnalytics.

The HEK293 cells used for IF stainings were authenticated according to the manufacturer ATCC using morphology, karyotyping and PCR based approaches to confirm the identity and to exclude intra and interspecies contaminations. These include an assay to detect species specific variants of the cytochrome C oxidase I gene (COI analysis) to rule out interspecies contamination and short tandem repeat (STR) profiling to distinguish between individual human cell lines and rule out intraspecies contamination.

The HeLa and HEK293 cells used for pre-rRNA analysis shown in Fig. 5e were authenticated by short tandem repeat (STR) profiling.

\section{Mycoplasma contamination}

Commonly misidentified lines (See ICLAC register)

\section{All cells used in this study (HEK293T, HEK293, HeLa) were tested negative for mycoplasma contamination.}

No commonly misidentified cell lines were used in this study. 